Fritz Blakolmer

\title{
Der autochthone Stil der Schachtgräberperiode im bronzezeitlichen Griechenland als Zeugnis für eine mittelhelladische Bildkunst*
}

Stellte Robin Hägg 1997 in einem Artikel die provokante Frage »Did the Middle Helladic people have any religion? «1, um unseren schlechten Kenntnisstand der Mittelbronzezeit auf dem griechischen Festland plakativ auf den Punkt zu bringen, so lautete die Antwort selbstverständlich: Ja. Sie waren keine Atheisten! Anders verhält es sich bei der im vorliegenden Beitrag gestellten Frage: Gab es eine mittelhelladische Bildkunst? Hierbei wird man nicht automatisch an eine rhetorische Frage denken, sondern durchaus die Möglichkeit in Betracht ziehen, dass auf dem griechischen Festland in vormykenischer Zeit eine so gut wie gänzlich anikonische künstlerische Ausdrucksweise bestand, deren Motivdekor sich weitgehend auf Wellenbänder, Spiralen und Rosetten an Gefäßkörpern konzentrierte. So wurde in der Forschung an der Existenz einer mittelhelladischen Ikonographie vor dem Einsetzen massiven kretischen Einflusses in der Schachtgräberzeit in der Regel gezweifelt: »No Middle Helladic tradition is discernible beyond. [...] A principal difficulty seems to be the shift from non-pictorial imagery« (E. T. Vermeule ${ }^{2}$. »Features considered characteristic of mainland art in the Late Bronze Age [...] may have been acquired, learned or invented in the Shaft Grave period itself", während sich die Zeit davor als »practically devoid of anything that can properly constitute a tradition of fine art« präsentiert hätte, was bedeutete, dass »)Helladic purity< must be seriously questioned almost from the moment of its birth.« (J. Hurwit ${ }^{3}$ ). Lediglich W. Heurtley schloss aus der Ikonographie der Reliefstelen aus Schliemanns Gräberrund A auf eine »native tradition for the figurescenes« im schachtgräberzeitlichen Mykene ${ }^{4}$.

Es muss eingestanden werden, dass eine Erörterung dieses Themas eher virtuellen Charakter besitzt, beschränkt sich die Evidenz für eine mittelhelladische Figuralkunst doch vorerst auf einige wenige >Strichmännchen und schematische Tiermotive auf Keramikscherben aus Argos, Mykene, Tsoungiza, Korakou, Kirrha, Iolkos und Kolonna auf Ägina ${ }^{5}$. Diese bescheidenen Primärquellen lassen sich jedoch deutlich

\footnotetext{
* Für die Genehmigung des Studiums ausgewählter Stücke im Archäologischen Nationalmuseum in Athen möchte ich Nikolaos Kaltsas und Lena Papazoglou-Manioudaki danken. Für die kritische Durchsicht des Manuskriptes danke ich Hubert D. Szemethy und für die redaktionelle Betreuung Barbara Brandt. Überlegungen zum vorliegenden Thema erscheinen auch in folgenden Publikationen: F. Blakolmer, The Iconography of the Shaft Grave Period as Evidence for a Middle Helladic Tradition of Figurative Arts?, in: A. Philippa-Touchais u. a. (Hrsg.), MESOHELLADIKA. The Greek Mainland in the Middle Bronze Age. International Conference, Athens, 8-12 March 2006, BCH Suppl. (in Druck); F. Blakolmer, Gab es eine mittelhelladische Bildkunst?, in: G. Grabherr - B. Kainrath (Hrsg.), Akten des 11. Österreichischen Archäologentages in Innsbruck, 23.-25. März 2006, Ikarus 3 (Innsbrucker klassisch-archäologische Universitätsschriften) (Innsbruck 2008) 65-72.

${ }^{1}$ R. Hägg, Did the Middle Helladic people have any religion?, Kernos 10, 1997, 13-18. s. auch Rutter 1993, 794 mit Anm. 202.

2 Vermeule 1975, 25 bzw. 50 .

${ }^{3}$ Hurwit 1979, 415. s. weiters F. Matz, Kreta und frühes Griechenland (Baden-Baden 1975) 169; Dickinson 1977, 84; W.D. Niemeier, Zum Problem von Import und Imitation minoischer Keramik in frühmykenischer Zeit, in: Aux origines de l'hellénisme. La Crète et la Grèce, Festschrift Henri van Effenterre (Paris 1984) 113.

${ }^{4}$ Heurtley 1921-1923, 145.

${ }^{5}$ s. A. Furtwängler - G. Löschcke, Mykenische Vasen. Vorhellenische Tongefäße aus dem Gebiete des Mittelmeeres (Berlin 1886) 54 Nr. 169 Taf. 23; C. W. Blegen, Korakou. A Prehistoric Settlement near Corinth (Boston 1921) 24 Abb. 34, 2; D. A. Theocharis, Iolkos. Whence sailed the Argonauts, Archaeology 11, H. 1, 1958, Abb. auf S. 17; S. A. Immerwahr, Some pictorial fragments from Iolkos in the Volos Museum, AEphem 1985, 86 f. Abb. 1 a. b; L. Dor u. a., Kirrha. Étude de préhistoire
} 
erweitern, begeben wir uns auf die Suche nach nichtkretischen Aspekten in den schachtgräberzeitlichen Bilddenkmälern des 17./16. Jahrhunderts, unter denen sich eine Reihe lediglich >minoisierender Bildwerke festländischen Ursprungs befindet. Wurde in der bisherigen Forschung das helladische Idiom während der Schachtgräberzeit meist dadurch festgelegt, dass man von den spätmykenischen Palastperioden zeitlich zzurückblickte und im älteren Fundspektrum nach Analogien suchte ${ }^{6}$, so soll in diesem Beitrag ein anderer Weg beschritten werden. In der großen, künstlerisch heterogenen Menge von Bildwerken aus und über den Schachtgräbern von Mykene können wir heute zumindest ein Element klar definieren, und zwar die kretisch-minoische Bildkunst. Schachtgräberzeitliche Bildwerke, die Minoisches imitieren und adaptieren, erlauben folglich auf stilkritischem Wege ein Herausfiltern anderer, wahrscheinlich älterer, mittelhelladischer Züge. Ein solcher rautochthon-helladischer Anteik lässt sich somit im Ausschlussverfahren in Form nichtminoischer Elemente extrapolieren, definieren und möglicherweise einer weiter zurückreichenden Tradition des Mittelhelladikums zuweisen. Gerechtfertigt wird ein solches methodisches Procedere nicht zuletzt durch die Dekorornamentik aus dieser lebendigen Entwicklungsperiode des Umbruches im frühmykenischen Griechenland, die oft in vergleichbarer Weise einen hybriden Charakter erkennen lässt. Es sei vorausgeschickt, dass sich unter den Bildobjekten aus den Schachtgräbern von Mykene und anderen festländischen Fundkontexten des MH III-SH IIA m. E. noch viel mehr Beispiele kretischen Ursprungs befinden, als in der Forschung bisher angenommen wurde, und dass autochthon-helladische Stiltrends lediglich noch marginal in einigen Charakteristika von Thematik, Motivik, Komposition und Stil zum Ausdruck kommen. Lassen Sie mich daher die künstlerischen Eigenschaften der helladischen Bildtradition während der Schachtgräberzeit kurz vorstellen, bevor wir uns der konkreten Verständnisweise dieses festländischen $>$ Restelementes` zuwenden.

\section{Charakteristika der autochthon-helladischen Bildkunst der Schachtgräberzeit}

$\mathrm{Zu}$ nennen ist zunächst die Anordnung der Ornamentik und Bildmotive in breiten horizontalen wie auch vertikalen Paneelen wie auf den Goldplaketten der Holzschatulle aus Schachtgrab V (Abb. 2) und den meisten Steinreliefstelen in Mykene (Abb. 7-11. 21-22), wobei wir teils an den Aufbau mittelhelladischen Gefäßdekors erinnert werden? . Mehrere Bildstelen (Abb. 8. 11) lassen eine Vorliebe für breite Einfassungszonen um die Figurenszenen, d. h. eine bemerkenswert raktiveく Rolle der Bildrahmung, beobachten. Bild- und Ornamentfelder werden oft wie gleich gewichtete und gleichwertige Gestaltungselemente behandelt. Dies gilt für manche Reliefstelen (Abb. 7. 9. 10) ebenso wie für die Wandungen der genannten Holzschatulle (Abb. 2) und den Kammgriff aus Schachtgrab III, auf dessen Tierfries an der einen Seite (Abb. 3) mit einem Rosettendekor auf der Rückseite geantwortet wird ${ }^{8}$. Die großformatigen Spiral- und Mäanderornamente auf den Schachtgräberstelen (Abb. 7-11. 22) lassen eine gewisse Tendenz zur Monumentalisierung erkennen, dürften doch manche Dekormotive den kleinformatigen Metalltreibarbeiten entnommen worden sein. Auch die in einem Horror Vacui wurzelnde Kombination aus figürlicher Szenerie und abstrakter Dekorornamentik, etwa bei der Einbeziehung von Spiralmotiven als Füllung von Bildszenen auf Reliefstelen (Abb. 7. 9), sticht ins Auge.

Verblüffend sind oft die Ungeschicktheit bei der Konzeption der Gestaltungsfläche sowie der bemerkenswert ungezwungene und konzeptlose Umgang mit dem Regelmaß. Mehrere Spiralrapportfelder zeugen von sorgloser Improvisation, Beliebigkeit und geringem Qualitätsanspruch ${ }^{9}$ (Abb. 9, 10. 22), wie dies auch auf

phocienne (Paris 1960) 73 Taf. 32 a; R. J. Buck, Middle Helladic mattpainted pottery, Hesperia 33, 1964, 277 (Motive 130. 132-133) Taf. 44; E. Protonotariou-Deilaki, Ship representations from prehistoric Argolid (MH period), in: H. Tzalas (Hrsg.), Tropis II. 2nd International Symposium on Ship Construction in Antiquity, Delphi, 27, 28, 29 August 1987 (Athen 1991) 123-126; H. B. Siedentopf, Mattbemalte Keramik der Mittleren Bronzezeit, Alt-Ägina 4, 2 (Mainz 1991) 55 Nr. 74 Taf. 14; 62 Nr. 158 Taf. 37; Farbtaf.; 62 Nr. 162 Taf. 38; Rutter 1993, 778 f. Abb. 14; 791 f. Abb. 17; A. Philippa-Touchais, Aperçu des céramiques mésohelladiques à décor peint de l'Aspis d'Argos, I. La céramique à peinture mate, BCH 126, 2002, 32-34 Nr. 94 Abb. 25. 26.

6 s. Hurwit 1979, 416.

7 So bereits Heurtley 1921-1923, 140 f. 144.

${ }^{8}$ s. Velsink 2003, 2 (C 1) Abb. 1; S. 18 f.

${ }^{9}$ Dies ist noch auf einem weiteren Stelenfragment zu beobachten: Younger 1997, 237 Nr. 12 a Taf. 92 a. 


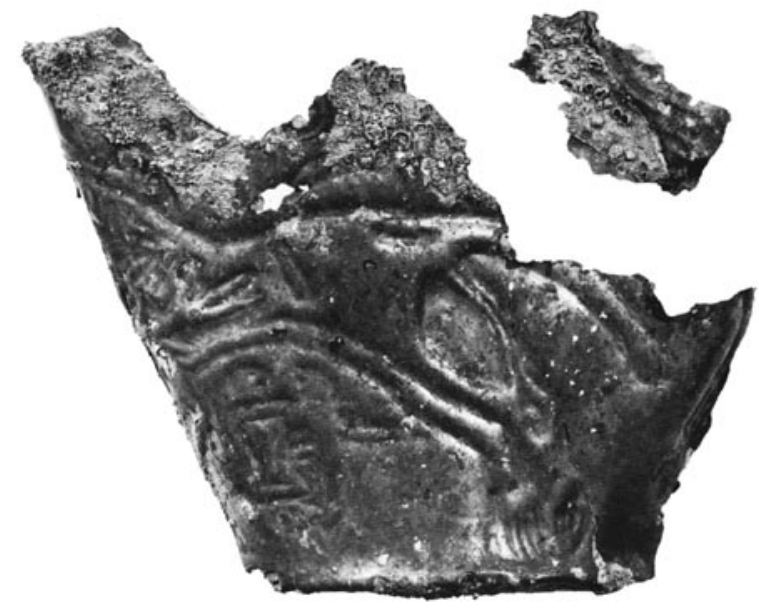

1 Silberbecher aus Schachtgrab Delta in Mykene

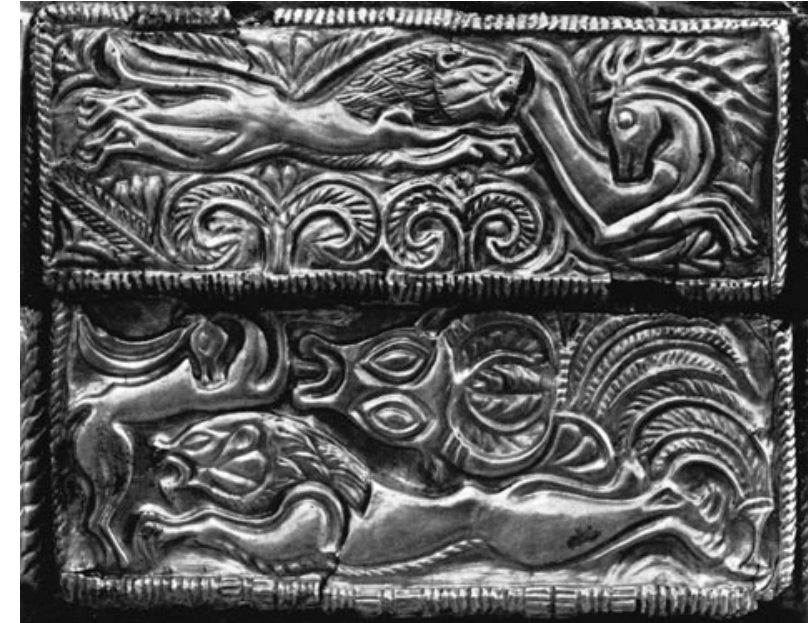

2 Goldplaketten aus Schachtgrab V in Mykene

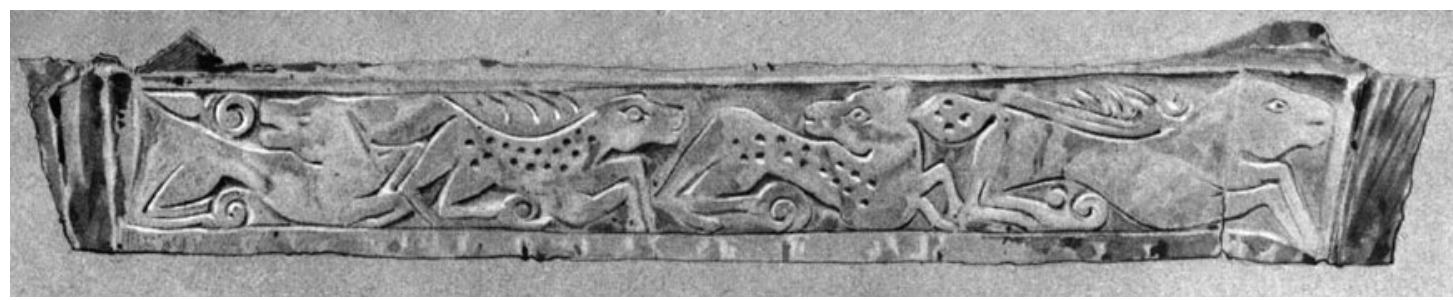

3 Goldblech aus Schachtgrab III in Mykene

manchen Golddiademen in Form von Asymmetrien zu beobachten ist ${ }^{10}$. Auch der zweifellos erst sekundär eingefügte Kampfgegner auf einer Bildstele (Abb. 10) veranschaulicht, dass der Sinn einer Vorzeichnung für diese festländischen Steinmetzen fremd und irrelevant war. Das weitreichende Fehlen eines kompositorischen Harmonieverständnisses bei komplexerer Ikonographie dokumentieren am besten die Goldappliken aus Schachtgrab V in Mykene (Abb. 2) mit ihrer Ansammlung kretischer Motive und dem frontalen Stierschädel als >kretisierendes` Dacapo. Der verschwindend kleine Jäger unter dem Löwenbauch auf dem Silberbecher aus Schachtgrab Delta (Abb. 1) zeugt von einer geradezu naiven Bildsprache, und dies könnte auch für die improvisiert verkleinerten Köpfe der Löwen auf dem Goldgoblet aus Schachtgrab V gelten ${ }^{11}$. Die Flügel der Greifen auf dem Nadelkopf aus Schachtgrab IV (Abb. 4) wurden auf ihre rankenförmigen Spiralhaken reduziert und lassen erkennen, wie fremd diese kretischen Figurenmotive dem festländischen Toreuten blieben. Grobheit und Schematismus in Konzeption sowie Ausführung kennzeichnen auch das Goldrelief mit Männerprozession aus Peristeria (Abb. 5) sowie die Goldscheibe mit Greif aus Thorikos (Abb. 6). Die unvollendete Steinstele über Schachtgrab Alpha in Mykene präsentiert eine so komplexe Ikonographie, dass es völlig rätselhaft bleiben muss, wie dieses minoische Tierüberfallmotiv ohne Reliefplastizität vom helladischen Steinmetzen hätte ausgeführt werden sollen ${ }^{12}$. Der Horror Vacui in der Komposition dominiert oft in einer Weise, dass die dichte, kompakte Anordnung der Bildelemente beim Erfassen des homogenen Bildgefüges stört ${ }^{13}$ (Abb. 2. 3. 6. 7. 9. 11), und diese Motiv- und Formendichte übersteigt

${ }^{10}$ Dazu bes. Karo 1930, 66. 71-73. 80 f. 184 f. Taf. 11-17. 35-41; B. Kling, Evidence for local style on the shaft grave diadems, in: Ph. P. Betancourt (Hrsg.), Temple University Aegean Symposium 6 (Philadelphia 1981) 29-38; Dickinson 1977, 74 Abb. 9 , 6.

${ }^{11}$ Zu diesem Goldgefäß s. Karo 1930, 125 f. Nr. 656 Taf. 126; Müller 1915, 312 f. Abb. 29; Marinatos - Hirmer 1973, Taf. 214 o.

12 s. Marinatos 1968, 175-177 Abb. 1; G. E. Mylonas, Ancient Mycenae. The Capital City of Agamemnon (Princeton, N. J. 1957)

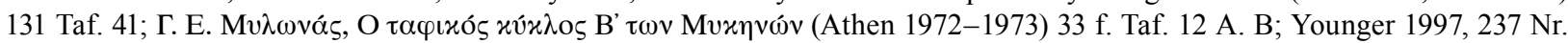
13 Taf. 89 b. c; 94 a.

13 Vgl. Vermeule 1975, 24: »concentration on the surface and not on the story«. 


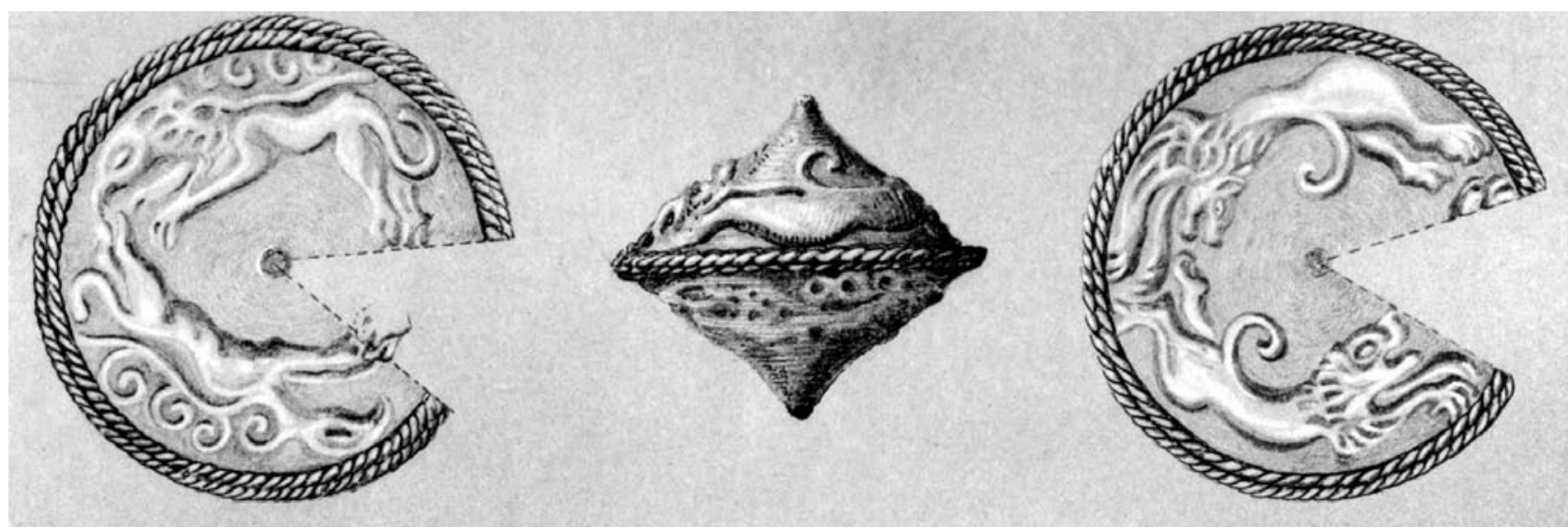

4 Goldener Nadelkopf aus Schachtgrab IV in Mykene

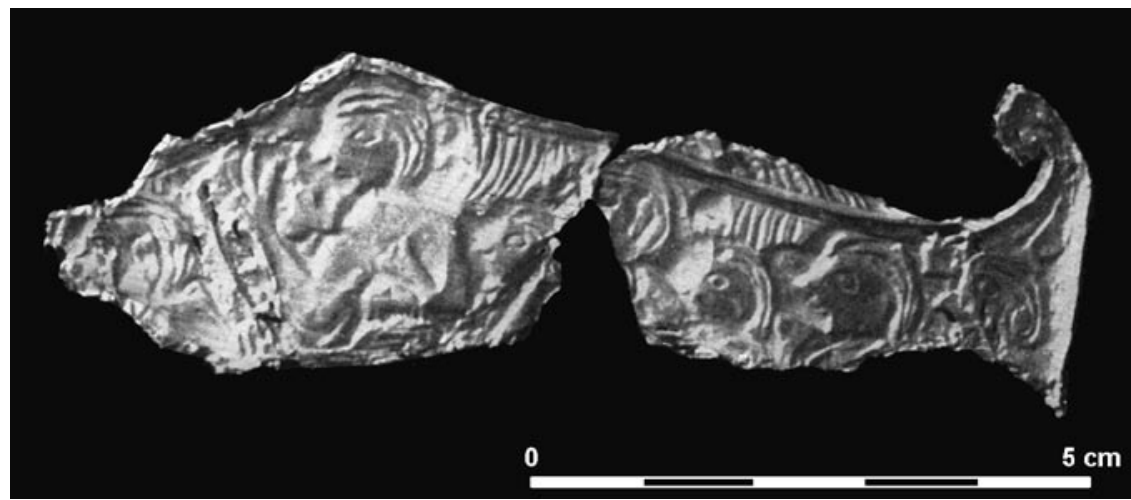

5 Goldblech aus Tholos 1 in Peristeria

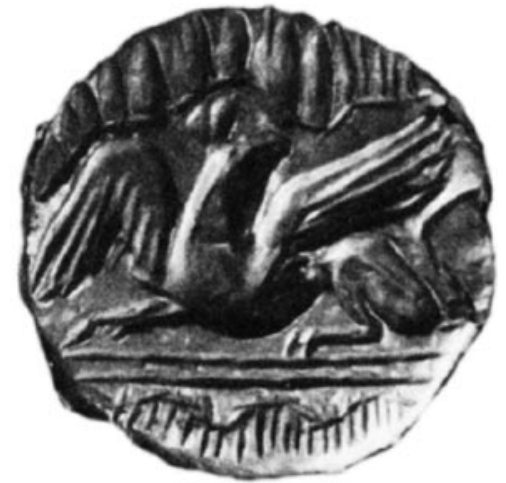

6 Goldblech aus Thorikos

deutlich das in der minoischen Bildgestaltung gängige Ausmaß. Eine Tendenz zur repetitiven Einförmigkeit der Motive lassen die Serien von Tierfiguren auf der >Pferde-Stele〈 (Abb. 8) und in stärker variierter Form auf dem Goldblech aus Schachtgrab III (Abb. 3) erkennen.

Bemerkenswert sind die flächenorientierten Reliefstile all dieser festländischen Bildwerke, sei es beim Gestalten in großformatigem Stein oder bei Metalltreibarbeiten mit einem Kern aus Stein, Elfenbein oder möglicherweise Holz. Flach, schemenhaft, betont graphisch und mit einem sun-minoischen schichtigkeit präsentieren sich Figurenmotive wie auch Ornamentik in unterschiedlichen Materialgattungen. Manche Metalltreibarbeiten lassen eine Splittung der Motive in abstrakte Einzelsegmente erkennen, eine Tendenz zur unorganischen Aufteilung der Formen und Figuren in kleinere, ovale Einzelfelder, wohl als Folge des ausgeprägten Horror Vacui ${ }^{14}$. Dies wird besonders deutlich im Nackenbereich der Löwen und Greifen auf dem goldenen Nadelkopf (Abb. 4), der Rumpfpartie des Löwen auf der Silbertasse (Abb. 1) wie auch an manchen Körperpartien der Tiere auf den Goldplaketten der sechseckigen Holzpyxis (Abb. 2). Diese Stileigenheit ist entweder graphischer Natur oder geht vielleicht eher auf die Reliefarbeit zurück. Anders verhält es sich bei den stilisierten, wulstförmigen Konturgraten der Tierfiguren in manchen Treibarbeiten (Abb. 1-2), die - ähnlich wie der >fliegende Galopp < - als signifikantes Element minoischer Kunst übernommen, oft in übertriebener, ’hyper-minoischer Weise angewandt und nicht selten auch inhaltlich missverstanden wurden.

Sehr frei ist der Umgang dieser festländischen Künstler mit den Gesetzen der Schwerkraft. Oft schweben die Figuren bindungslos und beliebig auf der Bildfläche (Abb. 7. 10), ohne dass wir dies auf das minoische Bildraumkonzept der `Kavaliersperspektive` zurückführen könnten. Die Mängel in der Maßstäblichkeit können eklatant sein, wie im Falle des kleinformatigen Jägers unter der Bauchwölbung des Löwen auf dem

${ }^{14}$ In der minoischen Bildkunst begegnet dies nur sehr selten. Vgl. z. B. die konkaven Tropfenformen in den Löwengesichtern der Dämonen auf dem Steinrhyton aus Malia: C. Baurain - P. Darcque, Un triton en pierre à Malia, BCH 107, 1983, bes. 36. 


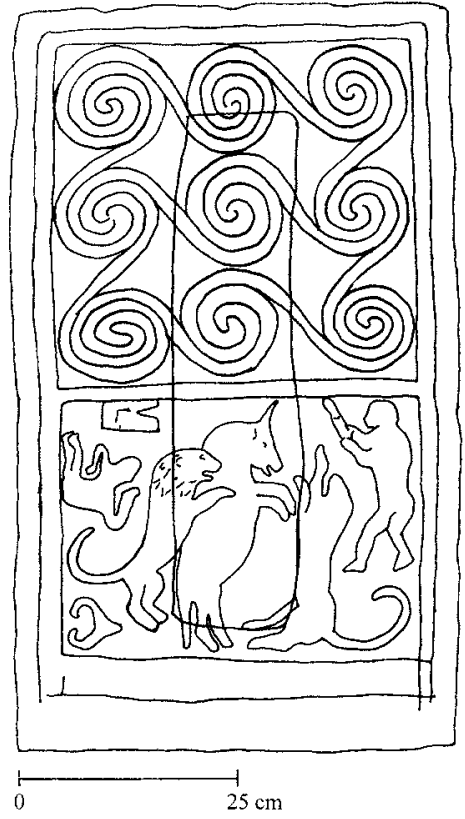

7 Reliefstele über Schachtgrab

Gamma in Mykene

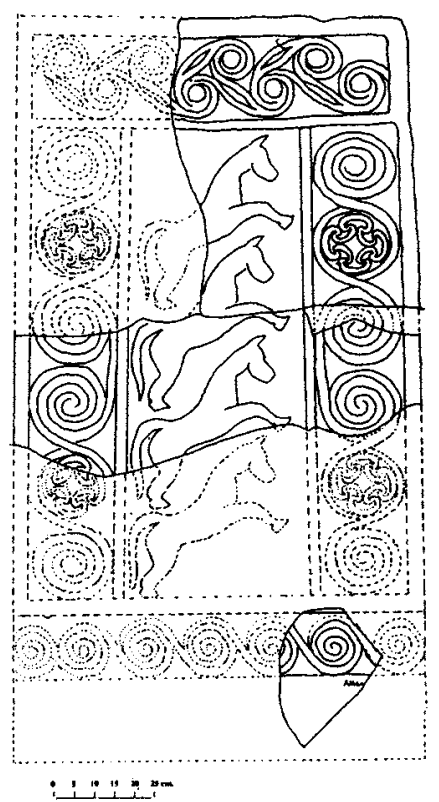

9 Reliefstele über Schachtgrab V in Mykene
8 Reliefstele aus Gräberrund $\mathrm{A}$ in Mykene
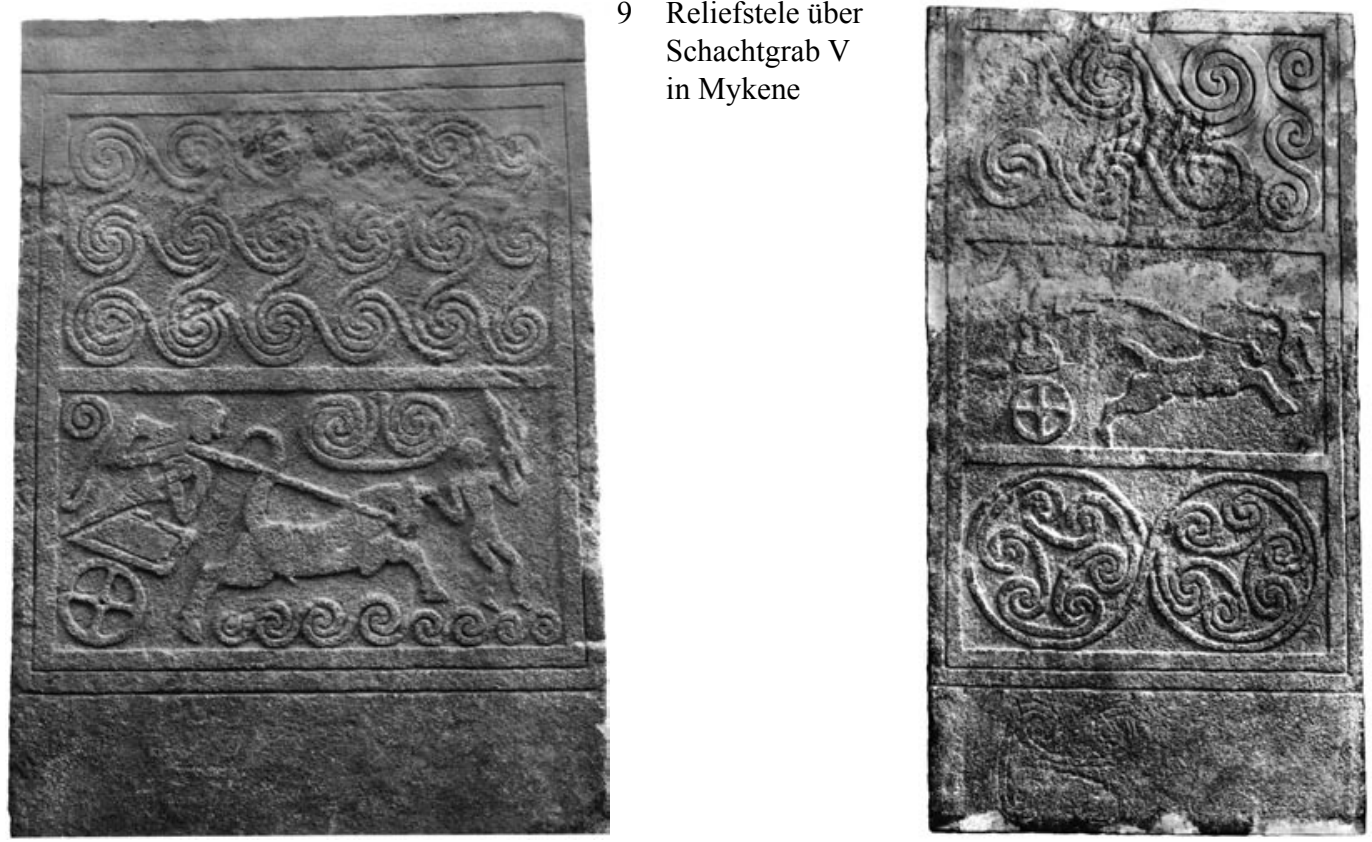

10 Reliefstele über Schachtgrab V in Mykene

Silberbecher aus Schachtgrab Delta (Abb. 1) oder des verkleinerten Horntieres auf einer Goldplakette aus Schachtgrab V (Abb. 2 u.). Eine für die Ägäis unübliche `Bedeutungsproportionierung` lässt der überdimensionierte Krieger im Wagen in einem Stelenbild erkennen (Abb. 9), und Anisokephalie prägt auch die Figurenreihe auf dem Goldblech aus Peristeria (Abb. 5). Von einer generellen künstlerischen Vernachlässigung der Bildgestaltung zeugen Inkonsistenzen in der Figurenproportionierung, etwa divergierende Längen von Vorder- und Hinterbeinen der Tiere, wie dies bei den Löwen auf dem goldenen Nadelkopf (Abb. 4) oder den Feliden auf der Kammverkleidung (Abb. 3) deutlich wird. Die Vorliebe für Spiral-, Schlaufen- und Schlingendekor kommt nicht nur in der schachtgräberzeitlichen Ornamentik deutlich zum Tragen, sondern teils sogar in der Figurengestaltung, wie beim manierierten Hirschgeweih auf Goldplaketten (Abb. 2) und den >Spiralranken` als Greifenflügel auf dem Nadelkopf (Abb. 4) - eine verspielt wirkende Ornamentalisierung und Geometrisierung von Details bzw. »traits of exaggerated fantasy«, in den Worten von E. Vermeule ${ }^{15}$.

\footnotetext{
${ }^{15}$ So das Urteil über die Greifenflügel von Vermeule 1975, 40. s. auch Karo 1930, 78.
} 


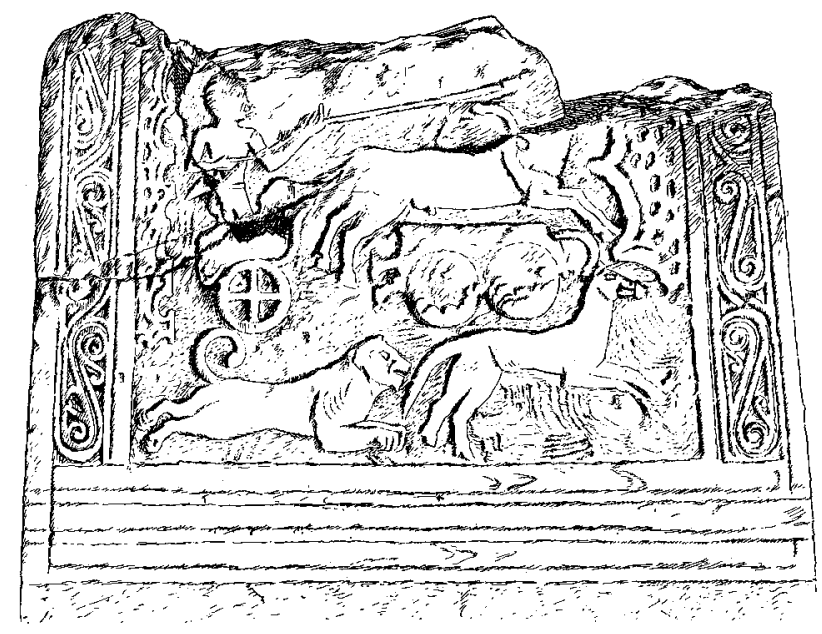

11 Reliefstele über Schachtgrab V in Mykene

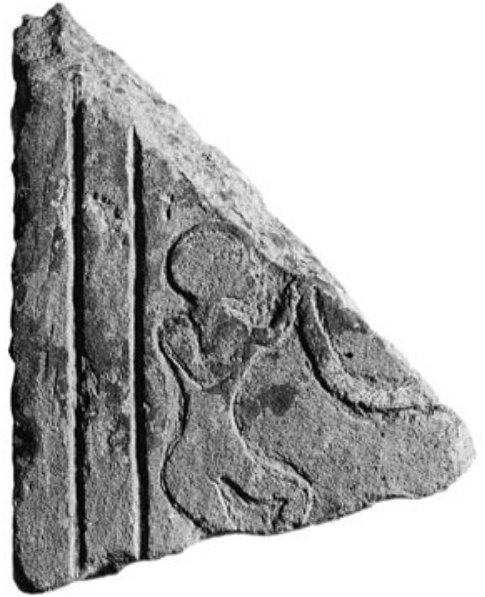

12 Fragment einer Reliefstele aus Gräberrund A in Mykene

Immer wieder bemerkenswert ist die Insignifikanz der Darstellungsform bei den unspezifischen Figurensilhouetten menschlicher Gestalten (Abb. 5. 7. 9-12). Die Variabilität in der Wiedergabe der Wagenkästen (Abb. 9-11) erlaubt uns keinerlei Urteil über ihre tatsächliche Form. Diesen Mangel an ikonographischer Konventionalisierung verdeutlichen auch die beiden additiv nebeneinander positionierten Räder eines zweifellos zweirädrigen Wagens auf einem Stelenfragment ${ }^{16}$.

Die menschlichen Figuren präsentieren sich meist schematisch, undifferenziert und mit minimaler Binnenzeichnung (Abb. 5. 7. 9-12. 21). Der bikonische Körper des kleinformatigen Jägers mit Pfeil und Bogen auf dem Silberbecher aus Schachtgrab Delta (Abb. 1) erinnert zwar ein wenig an die Menschenfiguren der mittelkykladischen Keramikmalerei ${ }^{17}$, doch haben diese Beispiele nur die schematische Grundstruktur gemeinsam. Die menschlichen Köpfe weisen gelegentlich ein scheiben- oder punktförmiges Auge und gekrümmte Haarsträhnen auf (Abb. 5. 12). Gerade die Figurenreihe auf dem Goldblech aus Peristeria (Abb. 5) veranschaulicht die Insignifikanz in der Darstellungsweise von Menschenköpfen. Im Gegensatz zu manchen Goldmasken aus den Schachtgräbern von Mykene ${ }^{18}$ besitzt in dieser festländischen Bildkunst keine einzige Männerfigur eine Bartangabe, was jedoch im summarischen Darstellungsstil begründet liegen könnte. Manche Jäger (Abb. 7) bzw. gegnerische Krieger (Abb. 9-11) auf den Stelenbildern sind meist in leichter Hocke wiedergegeben, und ihre abgeknickten Beine galten allem Anschein nach als Bildchiffre für rasche Bewegung. Als Charakteristikum gefallener Krieger dürfen wir die gänzlich unkoordinierten Gliedmaßen (Abb. 7) verstehen. Interessant ist der Umstand, dass die Arme mancher Figuren nicht in minoischer Manier nach außen abgelenkt, sondern über das Körperfeld geführt werden (Abb. 5. 7. 12).

Unterschiedliche Bewegungsschemata begegnen auch bei den Tiermotiven in diesen festländischen Bildern. Sehr bereitwillig erfolgte die Übernahme des minoischen >fliegenden Galopps (Abb. 2. 4. 11). Gänzlich anders präsentiert sich auf der Goldverkleidung des Kammgriffes aus Schachtgrab III der Habitus der vier Raubtiere (Abb. 3), die eher als laufend denn als kauernd zu verstehen sein dürften. Ein Spezifikum festländischer Bildwerke der Schachtgräberzeit bildet jedoch der `Absprunggalopp $<$ bei Pferden (Abb. 8-11), dem Hirsch auf der >plastischen Stele (Abb. 11), dem Löwen auf dem Silberbecher aus Schachtgrab Delta (Abb. 1) und einem der Greifen auf dem Nadelkopf aus Schachtgrab IV (Abb. 4). Dieses markante Laufschema besitzt keinerlei Parallelen in der übrigen ägäischen Ikonographie, sondern lediglich im Orient und findet auch in der späteren mykenischen Bildkunst keine Fortsetzung.

\footnotetext{
${ }^{16}$ Heurtley 1921-1923, 134-136 Nr. 9 a-b Abb. 30; Crouwel 1982, 74-76; Younger 1997, 236 f. Nr. 9 Taf. 92 a.

${ }^{17}$ Vgl. C. C. Edgar, The pottery, in: T. D. Atkinson u. a. (Hrsg.), Excavations at Phylakopi in Melos, Society for Promotion of Hellenic Studies Suppl. 4 (London 1904) 105 Taf. 13, 14. 17. 18.

${ }^{18}$ s. bes. G. Kopcke, Zum Stil der Schachtgräbermasken, AM 91, 1976, 1-13; O. Dickinson, The >face of Agamemnon<, Hesperia 74, 2005, 299-308
} 
Manche Stelenbilder des Gräberrundes B zeugen von einer gewissen Vorliebe für die Einbeziehung des Menschen, sogar in minoische Tierüberfallszenen (Abb. 7). Auch auf dem Silberbecher aus Schachtgrab Delta (Abb. 1) wurde das Motiv des laufenden Löwen durch einen Jäger bereichert. Eine additive Kombination unterschiedlicher Bildthemen dokumentiert am besten die patchworkartige Ikonographie der splastischen Steleく aus Mykene (Abb. 11), die ein festländisches Kampfbild mit einer >minoisierenden vereint; die flankierenden Felskorallenmotive wurden wahrscheinlich von einer kretischen Metalltreibarbeit mit maritimer Ikonographie entlehnt ${ }^{19}$. Eine vergleichbar inkohärente Kombination von $>$ Minoismen lassen auch die Goldplaketten der Holzpyxis aus Schachtgrab V (Abb. 2) erkennen. Nicht nur die Stelen der beiden Gräberrunde (Abb. 7. 9-12. 21), sondern auch der Silberbecher aus Schachtgrab Delta (Abb. 1) bezeugen eine prononcierte Präferenz der Themen Jagd und Kampf ${ }^{20}$ - eine Bildwelt der Tiere und der Männer. Keine einzige Darstellung einer Frau ist in dieser >autochthonen Kunst bisher bekannt. Das Bedürfnis, die Figurenhandlung in ihrem natürlichen Umfeld darzustellen, war kaum ausgeprägt. Wenn Pflanzen- und Terrainmotive begegnen, so resultieren diese stets aus der Nachahmung minoischer Vorbilder und dienen - wie auch manche Spiralmotive - meist primär dem Füllen der Freiräume. Dies gilt etwa für die Pflanzenmotive auf den Goldplaketten der Schatulle aus Schachtgrab V (Abb. 2), für das herabreichende >Rockworkı auf dem Goldblech aus Thorikos (Abb. 5) und in der Figurenszene aus Peristeria (Abb. 6), für die insignifikanten Ziselierungen auf dem Goldblech mit Tierfries aus Schachtgrab III (Abb. 3) sowie für die flankierenden Felskorallenmotive auf der >plastischen Stele< (Abb. 11).

\section{Das Verständnis des autochthonen Stils der Schachtgräberzeit}

Wie haben wir diese ca. 28 Spezifika der autochthon-festländischen `Schachtgräberkunstく konkret zu verstehen? Sie begegnen an mindestens 17 Bildwerken aus Mykene, Peristeria und Thorikos, und zwar in Treibarbeiten aus Gold und Silber, in geschnitztem Elfenbein sowie in Stein gemeißelt, fehlen jedoch auf Siegelsteinen und Goldringen sowie an Objekten aus Fayence und Ton. Die Menge der Beispiele, die Konzentration auf Werkstoffgattungen in meist verwandten Techniken und vor allem eine gewisse stilistische und ikonographische Homogenität der Bildsprache erlauben uns, von einer spezifischen Gruppe von künstlerischen Zeugnissen zu sprechen. Zwar könnte man einwenden, dass schematische, teils wenig signifikante Motive wie die genannten keinerlei Vergleich zuließen. Dem steht jedoch der Umstand entgegen, dass sich nicht nur die zeitgleiche neopalatiale Bildkunst Kretas und die jüngere des spätmykenischen Festlandes - selbst in ihren einfachsten Ausbildungen - deutlich davon unterscheiden, sondern auch die ebenso einfache figurale Bildwelt der mittelbronzezeitlichen Kykladen zeichnet sich größtenteils durch andere Eigenschaften aus ${ }^{21}$. Die genannten Charakteristika der festländischen Bildgestaltung können auch schwerlich als rein individuell oder gar als Spezialitäten einer einzigen Werkstatt verstanden werden. Für solche Erklärungsmodelle präsentieren sich Verteilung, Vielseitigkeit und Variabilität der Beispiele wiederum zu komplex und variantenreich. Manche dieser künstlerischen Züge erscheinen in einem Maße gefestigt, dass die Annahme naheliegt, hier die Reste einer tendenziell homogenen Kunstrichtung zu erkennen ${ }^{22}$. Und wenn wir in unsere Überlegungen den Schlaufen- und Wellenbanddekor einbeziehen, so besitzt diese festländische Kunst sogar eine beträchtliche geographische Ausdehnung, die weit über Mykene hinausreichte und sogar regionale Differenzierungen aufweisen dürfte ${ }^{23}$.

\footnotetext{
${ }_{19}$ Dazu auch Müller 1915, 287; A. Evans, The Shaft Graves and Bee-hive Tombs of Mycenae and their Interrelations (London 1929) 56; Evans 1935, 252; Mylonas 1951, 146 f.; Hood 1978, 99 f. mit Abb. 81.

${ }^{20}$ Den Beginn dieser festländischen Themenvorliebe erkannte hierin z. B. Vermeule 1975, 22-26.

${ }^{21}$ s. dazu u. Anm. 98.

${ }^{22}$ So auch Heurtley 1921-1923, bes. 145.

${ }^{23}$ Dazu K. Müller, Alt-Pylos II. Die Funde aus den Kuppelgräbern von Kakovatos, AM 34, 1909, 287; Penner 1998, bes. 139. 145 mit Abb. 29. Contra: O. Dickinson, »The Origins of Mycenaean Civilisation« revisited, in: R. Laffineur (Hrsg.), Transition Le monde égéen du Bronze moyen au Bronze récent. Actes de la deuxième Rencontre égéenne internationale de l’Université de Liège (18-20 avril 1988), Aegaeum 3 (Liège 1989) 132; J. H. Crouwel, Rezension von Penner 1998, AJA 105, 2001, 545.
} 
Zweifellos fassen wir in den vorgestellten schachtgräberzeitlichen Bildwerken den Charakter einer hybriden, stransitorischen< Kunst, und zwar in autochthoner Formensprache unter demonstrativer Miteinbeziehung minoischer Bildelemente, die eklektisch und in vielseitiger Form als Anregung verwendet wurden. Keines dieser Bildobjekte kommt gänzlich ohne kretisch-minoischen Einfluss aus und kann als rein autochthon-helladisch angesprochen werden. Der Silberbecher mit Löwenjagd (Abb. 1) beispielsweise verdankt zumindest seine Gefäßform der kretischen Tradition ${ }^{24}$, und selbst in Bildwerken wie der >Pferde-Steleく (Abb. 8) wurden minoische Ornamentmotive in die Rahmung integriert. Unübersehbar ist weiters, dass diese helladischen Künstler das technische Handwerk rascher erlernten als den Umgang mit dem sneuen Stik aus Kreta ${ }^{25}$. Dies bedeutet, dass die autochthon-festländischen Stilformen für uns lediglich noch im Stadium des Imitations- und Adaptionsprozesses des >Minoisierens〈 zum Vorschein kommen, und dies erschwert uns Definition und Verständnis des helladischen Elementes erheblich. Stets erkennen wir das Produkt eines kreativen Dialoges zwischen auf dem Autochthonen beruhenden Formvorstellungen des Festlandes und der fremden kretischen Kunst der `Neuen Zeit - Bildwerke, welche uns künstlerische Synkretismen in all ihrer individuellen Unschärfe vor Augen führen. Folgende Überlegung erscheint bei einem solchen künstlerischen Rezeptionsprozess wesentlich: Ein wirkliches Adaptieren von `Fremdem dann möglich, wenn bereits zuvor gewisse eigene Bildvorstellungen vorhanden waren. Für die Annahme, die festländischen Kunsthandwerker der Schachtgräberzeit lernten gänzlich aus dem Nichts heraus ${ }^{26}$, scheinen viele Charakteristika der Endprodukte jedenfalls zu gefestigt und konstant, sodass wir größtenteils von künstlerischen Adaptionen auf einer eigenständigen helladischen Basis sprechen wollen.

Das in den Altertumswissenschaften heute inflationär verwendete Schlagwort der >Akkulturation bildet in der Schachtgräberzeit unbestritten das nahezu omnipräsente Schlüsselphänomen bei der festländischen Auseinandersetzung mit kretischer Dekor- und Bildkunst während dieser Übergangsperiode. Umso bemerkenswerter ist es, dass bereits die Grabstelen des älteren Gräberrundes B (z. B. Abb. 7) kretisch-minoische Motivanleihen erkennen lassen ${ }^{27}$. Obgleich die relative Chronologie der Reliefstelen von Mykene unsicher bleiben muss, zeigen doch gerade die Stelenbilder der jüngsten Schachtgräber IV und V (Abb. 8-10) in geringstem Umfang kretische Elemente ${ }^{28}$. Dies muss keineswegs bedeuten, dass sich ein vermeintlich $>$ mittelhelladisches Element erst im Zuge der festländischen Auseinandersetzung mit kretischer Kunst herausbildete $^{29}$. Es wäre hingegen gut vorstellbar, dass die repräsentativen Semata im exklusiven Werkstoff Stein über den Schachtgräbern bewusst das `Eigene〈 präsentieren sollten. Aus diesen und weiteren Gründen erscheint es problematisch, a priori eine lineare Entwicklung in Richtung einer zielstrebigen \Minoisierung zu postulieren. Selbst die festländischen Reliefwerke aus SH IIB-IIIA1 lassen noch deutlich erkennen, dass dieser Lernprozess bemerkenswert langsam verlief und keineswegs streng unilinear vor sich gegangen sein dürfte.

Eine weitere Eigenschaft der hybriden helladischen Bildkunst der Schachtgräberzeit sticht uns ins Auge, und zwar ihr eklektischer Charakter bezüglich der Bildthemen. Zwar wurden offensichtlich minoische Tierjagdmotive und Tierlauf-Schemata aufgegriffen, nicht jedoch die so vielseitige Ikonographie der Frau und die reichhaltigen Pflanzenmotive der neopalatialen Bildwelt des minoischen Kreta, sieht man etwa von den Goldplaketten der Holzschatulle aus Schachtgrab V (Abb. 2) ab. Hinzu kommt das Phänomen, dass zwar Preziosen mit Kampfdarstellungen in minoischer Bildtradition, wie der Silberkrater aus Schachtgrab IV ${ }^{30}$

${ }^{24}$ Davis 1977, 46. 136 f. Nr. 30 Abb. 105; R. Laffineur, Les vases en métal précieux à l'époque mycénienne (Göteborg 1977) $10-17$.

${ }^{25}$ Vgl. dazu auch Müller 1915, 315 f.

26 So Dickinson 1977, bes. 85 .

${ }^{27}$ Dazu Pini 1985, bes. 165.

${ }^{28}$ Zur Konzentration des >Schlaufen- und Wellenbanddekors` in den jüngeren Schachtgräbern III, IV und V s. O. Dickinson, Invasion, migration and the Shaft Graves, BICS 43, 1999, 106.

${ }^{29}$ So z. B. Vermeule 1975, 25; Hurwit 1979, bes. 415.

${ }^{30}$ Karo 1930, 119 f. Nr. 605-607 Taf. 129-131; A. Sakellariou, Un cratère d'argent avec scène de bataille provenant de la IV ${ }^{\text {ème }}$ tombe de Mycènes, in: Atti e Memorie del $1^{\circ}$ Congresso Internazionale di Micenologia, Incunabula Graeca 25, 1 (Rom 1968) 262-265 Taf. 1-4; A. Sakellariou, Un cratère d'argent avec scène de bataille provenant de la IV ${ }^{\mathrm{e}}$ tombe de l'acropole de Mycènes, AntK 17, 1974, 3-20 Taf. 1. 2; Davis 1977, 225-229; F. Blakolmer, The silver Battle Krater from Shaft Grave IV at Mycenae: Evidence of fighting >heroes on Minoan palace walls at Knossos?, in: R. Laffineur - S. Morris (Hrsg.), EPOS. Reconsidering Greek Epic and Aegean Bronze Age Archaeology. 11th International Aegean Conference, UCLA, Los Angeles, 20-23 April 2006, Aegaeum 28 (Liège - Austin 2007) 213-224. 
oder die Fayence-Kanne aus Schachtgrab III ${ }^{31}$, am Fürstenhof von Mykene zur Disposition standen, für die Stelenbilder jedoch konsequent eine andere Ikonographie des Kriegers aufgegriffen wurde (Abb. 9-11), die, wie wir später noch sehen werden, eine gewisse Affinität zur nahöstlichen Ikonographie besitzen dürfte. Mit thematischen Präferenzen allein können wir diese festländische Bildkunst der Schachtgräberzeit und ihr eigenwilliges Rezeptionsverhalten offensichtlich nicht erklären.

Bemerkenswert ist einerseits die Aufgeschlossenheit der helladischen Künstler der Schachtgräberzeit und ihre Bereitschaft zur freien Rezeption zahlreicher Elemente der sneuen Kunst sches wurde in vielfältiger Hinsicht imitiert und adaptiert, und zwar allem Anschein nach ziellos, beliebig, oft ungeschickt und nicht selten in missverstandener Weise. Als Vorbilder dienten den festländischen Toreuten und Steinmetzen zweifellos importierte kretische Preziosen, die ihnen vor Ort zur Verfügung standen, und deren Zeitstil spiegelt sich gelegentlich auch in minoischen Bildwerken aus denselben Schachtgräbern wider $^{32}$. Andererseits weist $\mathrm{m}$. E. nichts auf >unbeholfene Künstler` (»a slightly clumsy artist ‘ $^{33}$ ) in Diensten zugewanderter minoischer >Meister hin: Was diesen helladischen Künstlern offensichtlich fehlte, waren kretische Kollegen, die ihnen die fremden minoischen Motive, den minoischen Stil und minoische Darstellungskonventionen vor Ort erklärten und sie vor formalen und inhaltlichen Missverständnissen bewahrten. Allem Anschein nach begnügten sich auch die schachtgräberzeitlichen Fürsten teils mit irgendetwas beliebig $>$ Minoisierendem<. Diese >plumpen ‘ festländischen Imitationen kretischer Motivik auf Preziosen begegnen in sepulkralen Kontexten jedenfalls Seite an Seite mit minoischen Originalen. Dies bedeutet, dass der Entstehungsprozess dieser festländischen Reliefbilder an elitären Prestigeobjekten allem Anschein nach ohne den direkten Eingriff einer >leitenden minoischen Hand erfolgte, und dies lässt uns an der Präsenz kretischer Künstler an schachtgräberzeitlichen Fürstenhöfen wie in Mykene grundsätzlich zweifeln.

\section{Eurasische Steppenkunst?}

Wie verhält es sich vor diesem künstlerischen Hintergrund der Schachtgräberzeit mit der in der Forschung öfters geäußerten Annahme >eurasischer Zuwanderer aus dem Norden ${ }^{34}$ ? Der vorliegende Figuralstil begegnet auf den Steinreliefstelen zwar gemeinsam mit Wellenbändern, Mäandermotiven, Volutendekor und komplexen Spiralkompositionen (Abb. 7-11. 21-22), doch dürfen diese Ornamentmotive nicht mit der markanten Dekorweise des `Schleifen- und Schlingenstils` auf schachtgräberzeitlichen Goldplaketten, Beinknöpfen mit Goldüberzug, vereinzeltem Goldzierat an Schwertgriffen etc. verwechselt werden. Während letztgenannte Ornamentstrukturen in der Tat eine unverkennbare Ähnlichkeit mit einer entsprechenden Formensprache auf dem Balkan und in entfernteren Regionen erkennen lassen ${ }^{35}$, erweisen sich die improvisiert kombinierten Ornamente auf den Schachtgräberstelen m. E. oft als eigenwillig >minoisierendeく Dekormotive mit unverkennbar kretischen Wurzeln ${ }^{36}$ und sollten in die Diskussion der direkten Kontakte zwischen

${ }^{31}$ Müller 1915, 332; G. Karo, Die Schachtgräber von Mykenai, AM 40, 1915, 165 Taf. 20, 1; Karo 1930, 60 f. Taf. 23; Evans 1935 , 690. 868 Abb. 858; K. P. Foster, Aegean Faience of the Bronze Age (New Haven - London 1979) 124-126 Abb. 86 Taf. 33.

32 Vgl. z. B. die Darstellungsweise der Löwenmähnen und Bauchwülste an Felidenkörpern sowie das thematische und ikonographische Spektrum der Metallobjekte und Siegel aus den Schachtgräbern von Mykene.

33 So J. G. Younger, The Mycenae-Vapheio Lion Workshop, III, in: Ph. P. Betancourt (Hrsg.), Temple University Aegean Symposium 6 (Philadelphia 1981) 68

${ }^{34}$ Zum vermuteten >eurasischen` Einfluss s. J. Boehlau, Die Spirale in der Bandkeramik, PZ 19, 1928, 54-98; M. Meurer, Der Goldschmuck der mykenischen Schaftgräber, JdI 27, 1912, 208-227; O. Kandyba, S-spiral in the decoration of the DniestroDanubian Neolithic pottery, AJA 40, 1936, 228-245; M.-L. und H. Erlenmeyer, Kassitische Goldarbeiten aus dem Schachtgrab III in Mykene?, Kadmos 3, 1964, 177 f.; S. Piggott, Ancient Europe (Edinburgh 1965) 123-140; Vermeule 1975, 22-26; Dickinson 1977, 72-82; Kling (Anm. 10) 29-38; S. Diamant, Mycenaean origins: Infiltration from the North?, in: E. B. French - K. A. Wardle (Hrsg.), Problems in Greek Prehistory. Papers Presented at the Centenary Conference of the British School of Archaeology at Athens, Manchester, April 1986 (Bristol 1988) bes. 157; Penner 1998, bes. 136-151 (s. dazu die Rezensionen von E. Kaiser, PZ 75, 2000, 239-241; J. H. Crouwel, AJA 105, 2001, 545 f.; W. David, Germania 79, 2001, 443-448); J. Makkay, The Early Mycenaean Rulers and the Contemporary Early Iranians of the Northeast (Budapest 2000) 22-25.

${ }^{35}$ Besonders überzeugend ist die umsichtige Argumentation von David 2001, 51-80.

${ }^{36}$ Dazu bereits A. Evans, The Palace of Minos at Knossos II (London 1928) 195-202 Abb. 105-110 B; Walberg 1986, 39-56; J. L. Crowley, Geometry in Minoan design, in: R. Laffineur - Ph. P. Betancourt (Hrsg.), TEXNH. Craftsmen, Craftswomen 
dem schachtgräberzeitlichen Griechenland und dem >eurasischen Norden` nicht einbezogen werden. Zwar erinnern einige künstlerische Aspekte der autochthon-helladischen Bildwerke, wie Ornamentalisierung und Geometrisierung, Segmentierung der Motive und kurvilineare Formen, teils verblüffend an sog. eurasische Nomadenkunst ${ }^{37}$, doch erfuhr der vorliegende Stil seine konkrete Ausprägung am ehesten auf dem griechischen Festland selbst ${ }^{38}$. Von einem >eurasischen Tierstik in dieser Figuralkunst ${ }^{39}$ ist m. E. keine Rede, besitzen doch so manche Tiermotive mit ihren Körperkrümmungen, wie später noch gezeigt wird, deutliche Entsprechungen auf Kreta, und zwar teilweise bereits in Siegelbildern des früheren Mittelminoikums. Auch die schraubenförmige Körperhaltung von Hirsch und Ziegenbock auf den Goldplaketten aus Schachtgrab V (Abb. 2) darf keinesfalls als >eurasisches` Fremdelement angesprochen werden ${ }^{40}$, sondern bildet unzweifelhaft eine Motiventlehnung aus der zeitgleichen Bildkunst Kretas ${ }^{41}$. Wie zuvor erwähnt, begegnet bereits auf den frühen Schachtgräberstelen eine >minoisierendeく Mischkunst, wobei sich insbesondere die Tierüberfallmotive (Abb. 7) als eindeutig kretischen Ursprungs definieren lassen ${ }^{42}$. Dies gilt auch für manche Ornamente $^{43}$, wie etwa den >flachen Mäander` (Abb. 20-23), wie wir später noch sehen werden. Eine Klärung der Frage, ob der $>$ Schleifen- und Schlingenstik der schachtgräberzeitlichen Ornamentik auf Zuwanderer oder Importe aus den >eurasischen Steppenregionen in die festlandgriechischen Zentren zurückgeführt werden kann oder eine weiter zurückreichende lokale Tradition darstellt, kann hier nicht geboten werden, doch scheint aus mehreren Gründen die Annahme einer autochthon-helladischen Verbreitung im Moment am plausibelsten ${ }^{44}$. Im Hinblick auf Ikonographie und Ornamentik der Kunstdenkmäler der Schachtgräberzeit lässt sich daher m. E. neben dem autochthon-festländischen und dem kretisch-minoischen Element keine dritte wesentliche künstlerische Traditionslinie überzeugend voraussetzen.

\section{Entlehnung ägyptischer Bildmotive?}

Obgleich wir die vereinzelten >Aigyptiaka in den Schachtgräbern von Mykene wohl größtenteils auf eine Vermittlung durch Kreta zurückführen können ${ }^{45}$, dürfte in der Bildkunst dennoch eine Ausnahme erkennbar sein. Das vor allem in Wagenkampfszenen beliebte Laufschema der Pferde in einer Art `Absprunggalopp< besitzt möglicherweise Wurzeln in der nahöstlichen Ikonographie. Ein hilfreiches Indiz für seine regionale Herleitung könnte das Detail bilden, dass der Hinterhuf meist nur mit der Spitze den Boden berührt (Abb. 9-10), was auf eine Entlehnung aus der ägyptischen Bildkunst hinweisen dürfte ${ }^{46}$. Nicht der Streitwagen

and Craftsmanship in the Aegean Bronze Age. Proceedings of the 6th International Aegean Conference, Philadelphia, Temple University, 18-21 April 1996, Aegaeum 16, 1 (Liège - Austin 1997) 81-92; Pini 2004.

37 Dazu bereits Chapouthier 1938, 43 f. Abb. 15 b; 16 a. b; Vermeule 1975, 40. Vgl. E. C. Bunker, Nomadic Art of the Eastern Eurasian Steppes (New York 2002) 18 Abb. 22; 27 Abb. 36; 27 f. Abb. 36. 40; 29 Abb. 41; 62 f. mit Abb.; 108 f. mit Abb.; 158-160 mit Abb.; 164 mit Abb.

38 s. bes. die Diskussion bei David 2001, 51-80.

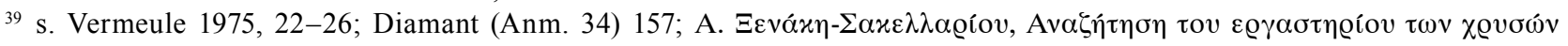

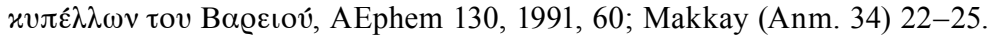

40 So P. Demargne, Naissance de l'art grec (Paris 1964) 185; Laffineur (Anm. 24) 64.

${ }^{41}$ Vgl. CMS II 7 Nr. 62; II 8 Nr. 235; VII Nr. 35; Evans 1921, 274 f. Abb. 204 s; Evans 1935, 255.

${ }^{42}$ s. Pini $1985,165$.

${ }^{43}$ Evans 1921, 195-202 Abb. 105-110 B; Evans 1935, 255; Heurtley 1921-1923, bes. 144 f.; Walberg 1986, 39-56; J.-C. Poursat, Ivory relief carving in Minoan Crete (2000-1450 BC), in: J. L. Fitton (Hrsg.), Ivory in Greece and the Eastern Mediterranean from the Bronze Age to the Hellenistic Period, British Museum Occasional Paper 85 (London 1992) 3-5 Taf. 1; Pini 2004, bes. 606 mit Hinweisen auf ältere Lit. s. auch S. M. Peirce, Syrian influence on Middle Minoan art, AJA 84, 1980, 226. So auch Vermeule 1975, 32 mit Abb. 37. 38: „Crete is not always as flowing and organized as we think.« Ähnlich auch J. L. Crowley, Essay on ten precious gems: Originality in Aegean art, in: Ph. P. Betancourt u. a. (Hrsg.), MELETEMATA. Studies in Aegean Archaeology Presented to Malcolm H. Wiener, Aegaeum 20, 1 (Liège - Austin 1999) 160. Zur Ähnlichkeit dieser Ornamentik mit jener in Kültepe und Bogazköy s. B. Kull, Untersuchungen zur Mittelbronzezeit in der Türkei und ihrer Bedeutung für die absolute Datierung der europäischen Bronzezeit, PZ 64, H. 1, 1989, 48-73.

${ }^{44}$ Vgl. auch die Überlegungen von David 2001, bes. 66-68.

45 s. S. Hiller, Die Schachtgräber von Mykene: in ägyptischem Kontext, Minos 29/30, 1994/1995, 7-31 bes. 20 f. 27. Anders R. Laffineur, Interconnections in the Aegean and the Eastern Mediterranean in the Shaft Grave period, BICS 40, $1995,247 \mathrm{f}$.

${ }^{46}$ Dazu bes. R. M. Czichon, Die Gestaltungsprinzipien der neuassyrischen Flachbildkunst und ihre Entwicklung vom 9. zum 7. Jahrhundert v. Chr. (München - Wien 1992) 33. 
selbst $^{47}$, sehr wohl aber die ikonographischen Motivtopoi des Wagenkampfes (Abb. 9-12 und 21) könnten auf eine direkte helladische Ableitung von ägyptischen Bildwerken zurückgehen ${ }^{48}$. Dies betrifft das Motiv des Kriegers im Pferdegespann und seines gegnerischen Fußsoldaten, das Motiv des gefallenen Gegners unter dem Gespann und vor allem jenes der Gespannpferde im >Absprunggalopp « mit ihrer signifikanten Hufstellung. Letztgenanntes Laufschema begegnet in Mykene nicht nur bei Pferdemotiven (Abb. 8-11), sondern auch bei dem Hirsch auf der splastischen Stele< über Schachtgrab V (Abb. 11) sowie dem Löwen auf dem Silberbecher aus Schachtgrab Delta (Abb. 1) und darf wohl am ehesten als individuelle helladische Motivübertragung von Gespannpferden auf weitere Tierarten betrachtet werden. Es erscheint weiters bemerkenswert, dass auf der Bildstele mit einer Reihe ungezäumter Pferde (Abb. 8) die Hufe anders positioniert wurden als bei den Wagengespannen; d. h., je weiter sich der helladische Künstler vom nahöstlichen Motivvorbild entfernte, desto freier ist auch sein Umgang mit motivischen Details. Es sticht auch ins Auge, dass bei diesen rägyptisierenden ২ Pferden die paarweise wiedergegebenen Beine nicht getrennt wurden, $d$. $h$. anders wiedergegeben wurden als bei den >minoisierenden Vierbeinern im Laufschema des >fliegenden Galopps (Abb. 2. 4. 11). Vielsagend ist darüber hinaus das Faktum, dass in der ägäischen Bildkunst die Motivvarianten des Pferdes bzw. des Wagengespannes auf den Grabstelen in Mykene ein isoliertes und kurzlebiges Phänomen der Schachtgräberzeit bleiben. Grundlegend anders präsentieren sich die laufenden Pferde auf der Schwertklinge aus Grab V ${ }^{49}$, das Wagengespann auf dem Goldring mit Hirschjagd aus Grab $\mathrm{IV}^{50}$, aber auch die kretischen Wagenmotive mit trabenden Pferden auf zeitgleichen Siegelabdrücken aus Kreta und Thera ${ }^{51}$ sowie die festländischen mykenischen Gespanndarstellungen jüngerer Zeit ${ }^{52}$. Lediglich der Wagen des Löwengespannes auf dem Amethyst-Rollsiegel aus der SH IIA-zeitlichen Tholos von Kazarma (Abb. 26) könnte noch eine gewisse Motivverwandtschaft mit den Beispielen der Stelenbilder aufweisen ${ }^{53}$. Dies bedeutet, dass wir im Falle der vorliegenden Ikonographie des Kriegers im Wagen am ehesten eine eigenständige helladische Imitation ägyptischer Motive erkennen können. Dieser künstlerische Impuls muss keineswegs auf einen intensiveren oder direkten Kontakt zwischen dem schachtgräberzeitlichen Mykene und dem Ägypten der zweiten Zwischenzeit oder der frühen 18. Dynastie schließen lassen. Zur Herausbildung dieser kurzlebigen Motivvariante im SH I-zeitlichen Mykene hätten vermutlich auch vereinzelte Bildszenen auf importierten ägyptischen Preziosen ausgereicht, die als stimulierend und adaptierenswert empfunden, d. h., in ähnlicher Weise verwertet wurden wie auch die kretisch-minoischen Vorbilder.

\footnotetext{
${ }^{47}$ Dazu bes. M. A. Littauer, The military use of the chariot in the Aegean in the Late Bronze Age, AJA 76, 1972, 145-157; Crouwel 1982; J. L. Crouwel, Rezension von Penner 1998, AJA 105, 2001, 545 f.; J. L. Crouwel, Early chariots in the Aegean and their Eastern connections, in: R. Laffineur - E. Greco (Hrsg.), EMPORIA. Aegeans in the Central and Eastern Mediterranean. Proceedings of the 10th International Aegean Conference, Athens, Italian School of Archaeology, 14-18 April 2004, Aegaeum 25, 1 (Liège - Austin 2005) 39-44. Zu dieser Diskussion s. ferner etwa W. Messerschmidt, Der ägäische Streitwagen und seine Beziehungen zum Nordeurasisch-vorderasiatischen Raum, ActaPraehistA 20, 1988, 31-44; Dickinson (Anm.

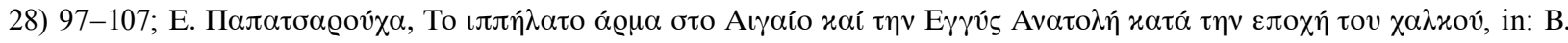

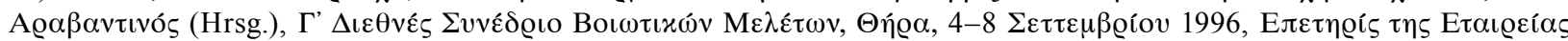

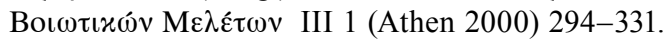

48 Vgl. Vermeule 1975, 16 f.; Hood 1978, 99; M. A. Littauer - J. H. Crouwel, Wheeled Vehicles and Ridden Animals in the Ancient Near East (Leiden 1979) 62 f. Abb. 33; Crouwel 1982, 119 f.; S. Hiller, Scenes of warfare and combat in the arts of Aegean Late Bronze Age. Reflections on typology and development, in: R. Laffineur (Hrsg.), POLEMOS. Le contexte guerrier en Égée à l'âge du Bronze, Actes de la $7^{\circledR}$ Rencontre égéenne internationale, Université de Liège, 14-17 avril 1998, Aegaeum 19, 2 (Liège - Austin 1999) 326 Taf. 71, 12. 13; S. Hiller, Beni Hassan, die Anfänge der minoischen Freskenkunst und Fragen der Chronologie, in: M. Bietak (Hrsg.), The Synchronisation of Civilisations in the Eastern Mediterranean in the Second Millennium B.C., II. Proceedings of the SCIEM 2000 - EuroConference, Haindorf 2nd of May - 7th of May 2001, DenkschrWien Gesamtakademie 29 (Wien 2003) 496 f. Abb. 22. 23.

${ }^{49}$ Karo 1930, 136 Nr. 748 Abb. 52. Taf. 80. 86 1.; Evans 1935, 831 f. Abb. 812; D. N. Tripathi, Bronzework of Mainland Greece from c. 2600 B.C. to c. 1450 B.C., SIMA Pocket-book 69 (Göteborg 1988) 286 Nr. 566 Taf. 66.

${ }^{50}$ CMS I Nr. 15; Crouwel 1982, 121 f. Taf. 10. Vgl. nun auch die Abdrücke eines SM I-Siegelringes mit von Greifen im >fliegenden Galopp gezogenem Wagen aus Knossos: CMS II 8 Nr. 193.

${ }^{51} \mathrm{Zu}$ diesen Siegelabdrücken aus Agia Triada, Sklavokampos und Akrotiri s. CMS II 6 Nr. 19 und 260; V Suppl. 3 Nr. 391.

52 s. Crouwel 1982, bes. 35-38; Ch. Morris, Design element analysis of Mycenaean chariot kraters: style, complexity and significance, in: E. Rystedt - B. Wells (Hrsg.), Pictorial Pursuits. Pictorial Painting on Mycenaean and Geometric Pottery. Papers from Two Seminars at the Swedish Institute at Athens in 1999 and 2001 (Stockholm 2006) 97-106. Vgl. weiters z. B. CMS V Suppl. 3 Nr. 244.

${ }^{53}$ CMS V Nr. 585; Crouwel 1982, 59 f. 158 (G 1) Taf. 9; Krzyszkowska 2005, 251 f. Abb. 481.
} 


\section{Zusammenhänge mit der Kunst der Altpalastzeit Kretas?}

Ungeachtet der minoischen Elemente, welche in diesen festländischen Bildwerken in Form vorbildhafter Ikonographie und Stilformen der neopalatialen Zeit (MM III-SM I) vielfach erkennbar sind, lohnt sich auch eine Prüfung der Frage, ob sich unter den autochthon-helladischen Motiven und Stilzügen mögliche Entsprechungen in der älteren Bildkunst Kretas, d. h. aus der Altpalastzeit (MM IB-MM IIB) oder gar den Perioden davor, finden lassen. Sollte die hier postulierte mittelhelladische Figuralkunst tatsächlich weiter als bis zum Beginn der Schachtgräberzeit zurückreichen, so können Anregungen aus der Bildkunst des FM II-MM II-zeitlichen Kreta keineswegs ausgeschlossen werden. Obwohl kulturelle Kontakte zwischen dem altpalatialen Kreta und dem mittelhelladischen Griechenland vor MH III bislang nur marginal bezeugt $\operatorname{sind}^{54}$, ist es umso bemerkenswerter, dass eine Reihe helladischer Stilzüge und Motive deutliche Parallelen in der kretischen Bildkunst erkennen lassen, und zwar in der einfachen, schematischen Ikonographie des Mittelminoikums vor dem Einsetzen des `Kretischen Naturalismus` im ausgehenden MM IIB und vor allem in $\mathrm{MM} \mathrm{III}^{55}$. Indizien dafür liefern uns in erster Linie ältere kretische Siegelbilder, die ab dem späten 3. Jahrtausend vereinzelt schematische menschliche Figuren in durchaus vergleichbarem Habitus erkennen lassen. So zeigt etwa ein vorpalastzeitliches Tonkonoid (FM III-MM IA) aus Archanes-Tourkogeitonia (Abb. 13) umgeben von einer Reihe von Löwen zwei schemenhafte männliche Gestalten ohne Binnengliederung und mit angewinkelten Gliedmaßen ${ }^{56}$. Trotz der zeitlichen Distanz ist die Ähnlichkeit etwa mit dem Figurenmotiv des Getöteten auf der Schachtgräberstele mit Löwe-Stier-Überfall in Mykene (Abb. 7) unübersehbar ${ }^{57}$. Obgleich die Beinstellung der leicht angewinkelten Knie von Figuren in Siegelbildern des altpalatialen Kreta oft mit einer Tätigkeit in hockender oder sitzender Haltung, etwa beim Töpfern, Melken oder Bogenschießen, zu erklären ist ${ }^{58}$, galt das Motiv des >unsicheren Standes` auf Kreta auch als allgemeine ikonographische Konvention für rasche Bewegung, wie dies etwa auf einem altpalastzeitlichen Siegelprisma aus der Werkstatt beim >Quartier Mu in Malia ${ }^{59}$ (Abb. 14) und einem Siegelkonoid aus Archanes-Phourni ${ }^{60}$ (Abb. 15) zu erkennen ist, und entspricht damit recht gut der Standardpose aktiver Fußkrieger auf den Bildstelen aus Mykene (Abb. 7. 9. 10. 12). Von einer Nacktheit darf sicherlich auch bei diesen menschlichen Figuren nicht gesprochen werden; vielmehr entsprach die undifferenzierte, silhouettenhafte Körperform der Stilsprache der frühkretischen Glyptik ebenso wie jener der Reliefstelen in Mykene. Erwähnt sei weiters, dass weibliche Figuren der kretischen Siegelglyptik bis MM III weitgehend fremd sind und auch diese ältere minoische Bildwelt primär eine Ikonographie der Männer und der Vierbeiner präsentiert ${ }^{61}$.

Die Tierposen bzw. das Laufschema der vier Feliden mit hoch emporgerecktem Rumpf auf dem Kammgriff aus Mykene (Abb. 3) erinnern zwar an ornamentalisierte Tiergestalten der >eurasischen Steppenkunst ${ }^{62}$,

${ }^{54}$ Dickinson 1977, 36 f.; J. B. Rutter, Stone vases and Minyan ware: A facet of Minoan influence on Middle Helladic Laconia, AJA 83, 1979, 464-469; Rutter 1993, 774 f.; P. Warren - V. Hankey, Aegean Bronze Age Chronology (Bristol 1987$) 68$ f.

55 Zur Kunst der Älteren Palastzeit Kretas s. J.-C. Poursat, Fouilles récentes à Mallia (Crète): L'art palatial minoen à l'époque de Camarès, GazBA 86, 1975, bes. 91-97; J.-C. Poursat, Iconographie minoenne: continuités et ruptures, in: P. Darcque - J.-C. Poursat (Hrsg.), L'iconographie minoenne. Actes de la table ronde d'Athènes (21-22 avril 1983), BCH Suppl. 11 (Paris 1985) 45 f.; S. A. Immerwahr, A possible influence of Egyptian art in the creation of Minoan wall painting, in: Darcque - Poursat a. O. 21-37; S. Immerwahr, Aegean Painting in the Bronze Age (University Park PA - London 1990) 21-37; Walberg 1986, 6-56; Wingerath 1995, 22-48; Blakolmer 1997, bes. 97-100; F. Blakolmer, The history of Middle Minoan wall painting: The \Kamares connection`, in: Betancourt u. a. (Anm. 43) 41-50; Krzyszkowska 2005, 85-98. 104-118.

${ }^{56}$ CMS II 6 Nr. 149 (zur Datierung: I. Pini, CMS II 6, S. 48); Y. Sakellarakis - E. Sakellaraki, Archanes. Minoan Crete in a New Light II (Athen 1997) 690 f. Abb. 7921.

${ }^{57} \mathrm{Zu}$ diesem Bildtopos s. allgemein Ch. G. Doumas, Conventions artistiques à Théra et dans la Méditerranée orientale à l'époque préhistorique, in: Darcque - Poursat (Anm. 55) 29-34; Ch. G. Doumas, Trade in the Aegean in the light of the Thera excavations, in: M. Marazzi - S. Tusa - L. Vagnetti (Hrsg.), Traffici micenei nel Mediterraneo. Problemi storici e documentazione archeologica, Atti del convegno di Palermo (11-12 maggio e 3-6 dicembre 1984) (Tarent 1986) $235 \mathrm{f}$.

58 s. z. B. CMS II 1 Nr. 300. 311. 385. 420. 442; II 2 Nr. 118 a. 303. 312; II 5 Nr. 326; II 8 Nr. 32. 33; Wingerath 1995 , $23-31$. Vgl. jedoch auch CMS II 8 Nr. 32.

${ }^{59}$ CMS II 2 Nr. 163 b.

${ }^{60}$ CMS II 6 Nr. 150; Sakellarakis - Sakellaraki (Anm. 56) 690 f. Abb. 792 r.

${ }^{61}$ Dazu auch Wingerath 1995, 41. 43.

${ }^{62}$ Vgl. Bunker (Anm. 37) 107 mit Abb. 


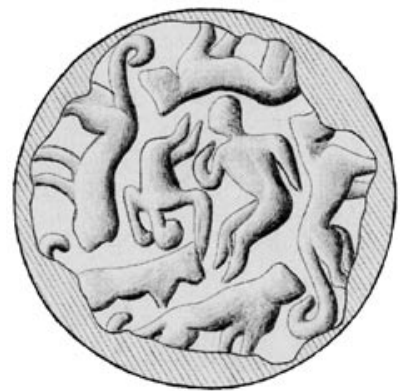

13 Siegel aus ArchanesTourkogeitonia

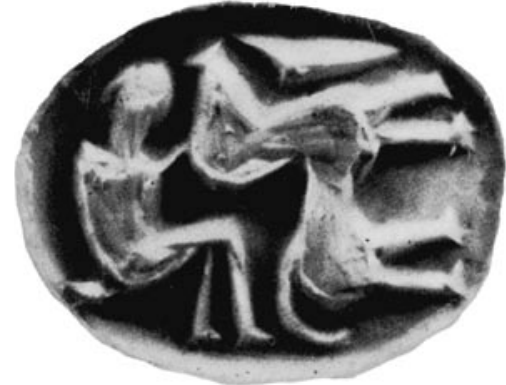

14 Siegel aus dem >Quartier Mu< in Malia

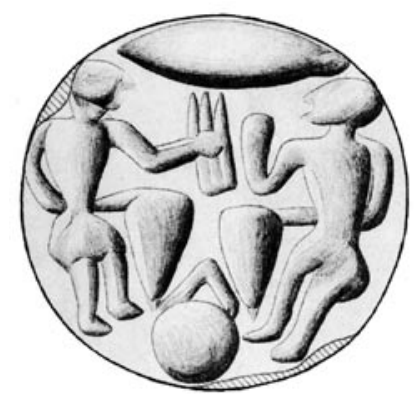

15 Siegel aus ArchanesPhourni

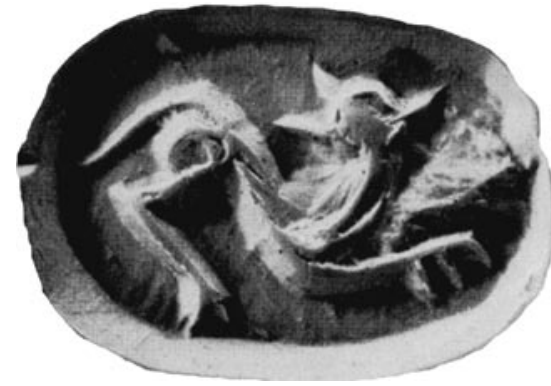

16 Siegel aus dem >Quartier Muく in Malia

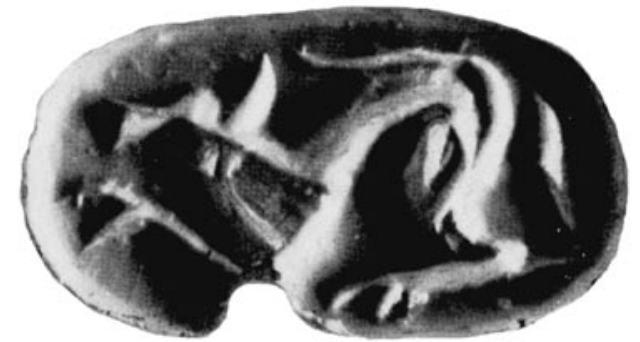

17 Siegel aus Potamies
18 Siegel aus Viannos
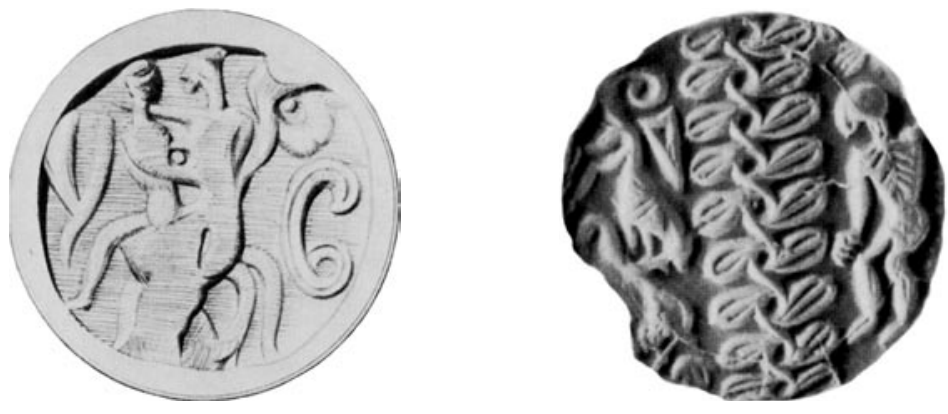

19 Siegel aus Marathokephalo

doch besitzt der S-förmig geschwungene Körper auch deutliche Entsprechungen in der frühen kretischen Siegelglyptik. Vierbeiner mit vergleichbarem Körperschwung bilden ein beliebtes, variantenreiches Motiv auf dreiseitigen Prismen von FM III-MM IIB ${ }^{63}$, wofür hier stellvertretend Beispiele aus der Siegelwerkstatt beim >Quartier Mu in Malia ${ }^{64}$ (Abb. 16) und aus dem zentralkretischen Potamies ${ }^{65}$ (Abb. 17) angeführt seien. Tendenzen einer markanten Segmentierung der Formen könnten aus der Levante nach Kreta gelangt $\operatorname{sein}^{66}$. Eine manieriert strukturierte Löwenmähne wie auf dem Nadelkopf aus Schachtgrab IV (Abb. 4) und

${ }^{63}$ s. CMS II 1 Nr. 408.427 c. 489 ; II 2 Nr. 78 c. 79 c. 82 b. 87 a. 89 a. 102 b. 113 c. 118 b. 122 a. 125 a. 126 a. 131 a. 139a. 161. 176 a. 218 b. 232 a. 234 a. 237 c. 245 c. 298 a. 303 b. 304 b; XII Nr. 31 a.

${ }^{64}$ CMS II 2 Nr. 139 a.

${ }^{65}$ CMS II 2 Nr. 218 b.

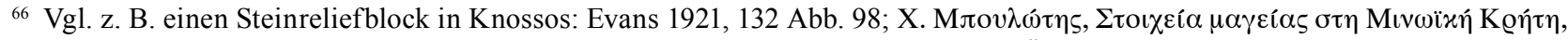

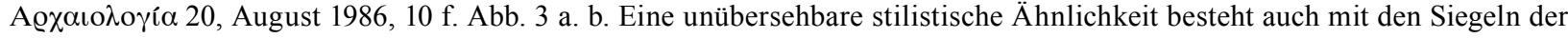
jüngeren sog. Lyre-player Group. Dazu E. Porada, A lyre player from Tarsus and his relations, in: S. W. Weinberg (Hrsg.), The Aegean and the East. Studies Presented to Hetty Goldman (New York 1956) 185-211; G. Buchner - J. Boardman, Seals from Ischia and the Lyre-player Group, JdI 81, 1966, 1-62. 


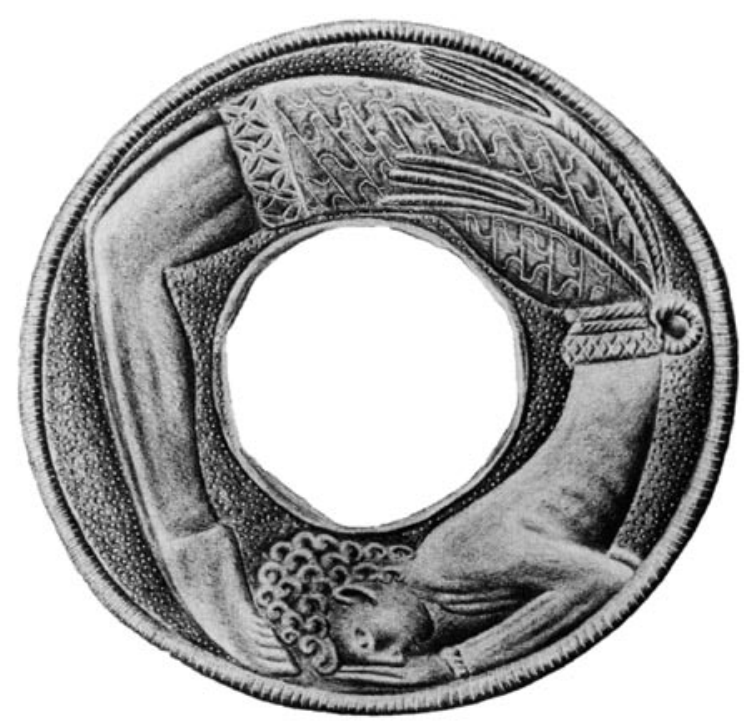

20 Goldscheibe mit Akrobat aus Malia
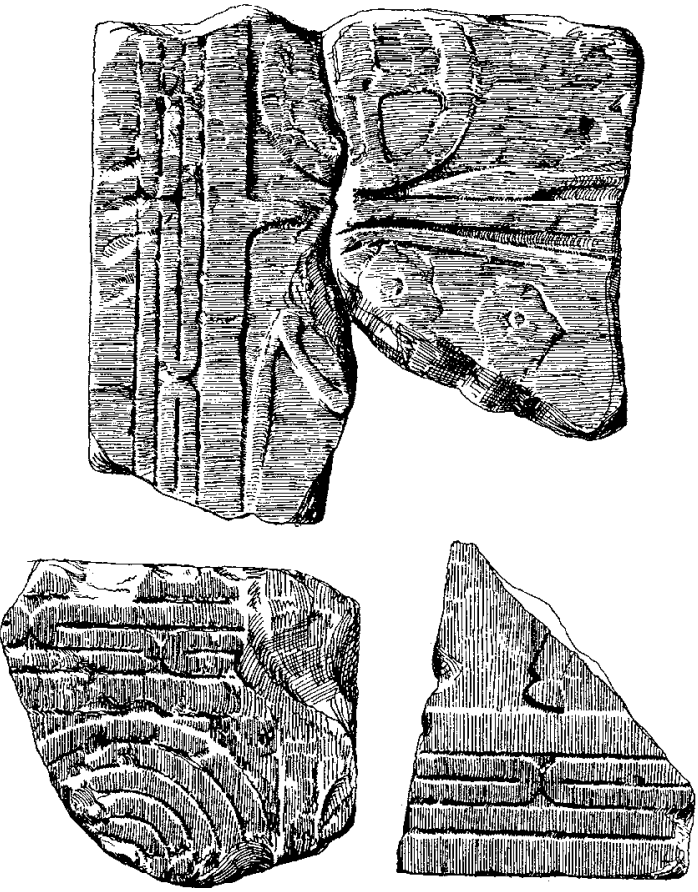

21 Fragmente einer Steinstele aus Gräberrund A in Mykene

vor allem auf dem Becher aus Schachtgrab Delta (Abb. 1) in Mykene begegnet auch bei Löwenmotiven der prä- und protopalatialen Siegelglyptik $\mathrm{Kretas}^{67}$ und wird erst allmählich bei naturalistischeren Löwen während MM II-III von Zackenformen abgelöst ${ }^{68}$. Ähnliches gilt für den in festländischen Bildkompositionen vorherrschenden Horror Vacui. Dieses Gestaltungsprinzip ist in der frühen Bildwelt Kretas deutlich stärker ausgeprägt als in der neopalatialen Ikonographie, und ein spiralähnliches, volutenförmiges Füllmotiv in einem Siegelbild aus der FM III-zeitlichen Nekropole von Viannos ${ }^{69}$ (Abb. 18) dokumentiert dieselbe Bereitschaft zur Einbeziehung komplexerer Füllornamentik in Figurenszenen, wie dies auf einer Reliefstele in Mykene der Fall ist (Abb. 9). Obgleich nur schwer vergleichbar mit den spiktorialisierten< Figuren der Kamares-Keramik ${ }^{70}$, wird doch auch in manchen festländischen Beispielen (Abb. 3-4) ein Hang zur Ornamentalisierung figürlicher Motive deutlich.

Das komplexe Bild eines elfenbeinernen Siegelzylinders aus der FM II-MM IB-zeitlichen Tholosgrabnekropole im zentralkretischen Marathokephalo (Abb. 19) präsentiert eine Reihe bereits genannter Gestaltungscharakteristika und ruft uns gleich mehrere Beispiele helladischer Bildwerke der Schachtgräberzeit ins Gedächtnis ${ }^{71}$. Die vermeintlich >nackteく Männerfigur mit schematischem Kopf weist die konventionell >hockende< Beinstellung auf, wofür wir weder ein Sitzmotiv noch den Platzmangel, sondern vielmehr die Verdeutlichung der Bewegtheit verantwortlich machen können. Als Jäger werden wir diese Gestalt eher nicht ansprechen wollen ${ }^{72}$, befindet sich auf der gegenüberliegenden, beschädigten Seite des Bildrundes doch

${ }^{67}$ s. z. B. CMS II 1 Nr. 224. 321. 497; II 5 Nr. 271-273; XII Nr. 8.

${ }^{68}$ Vgl. Siegelbilder aus dem >Archivio di cretule` in Phaistos: CMS II 5 Nr. 270 und 274. Zu Löwenmotiven in der frühägäischen Ikonographie s. allgemein J. Vanschoonwinkel, Les animaux dans l'art minoen, in: D. S. Reese (Hrsg.), Pleistocene and Holocene fauna of Crete and Its First Settlers, Monographs in World Archaeology 28 (Madison 1996) 362 f. 398-400; N. R. Thomas, The Early Mycenaean lion up to date, in: A. P. Chapin (Hrsg.), XAPI . Essays in Honor of Sara A. Immerwahr, Hesperia Suppl. 33 (Princeton, N. J. 2004) 161-206.

${ }^{69}$ CMS II 1 Nr. 446. Vgl. ähnliche Füllornamente in kretischen Siegelbildern: CMS II 5 Nr. 195; II 6 Nr. 190; V Suppl. 3 Nr. 476.

${ }^{70}$ G. Walberg, Kamares. A Study of the Character of Palatial Middle Minoan Pottery, Boreas 8 (Uppsala 1976) 65-69; G. Walberg, Provincial Middle Minoan Pottery (Mainz 1983) 57-61; Walberg 1986, 6-21; Blakolmer 1997, 102 f. s. weiters A. Furumark, The Mycenaean Pottery: Analysis and Classification (Stockholm 1941) 133-150.

${ }^{71}$ CMS II 1 Nr. 222 a; Galanakis 2005, 22. 31 Abb. 68, 1.

${ }^{72}$ Vgl. Krzyszkowska 2005, 67 mit Abb. 112 c. 


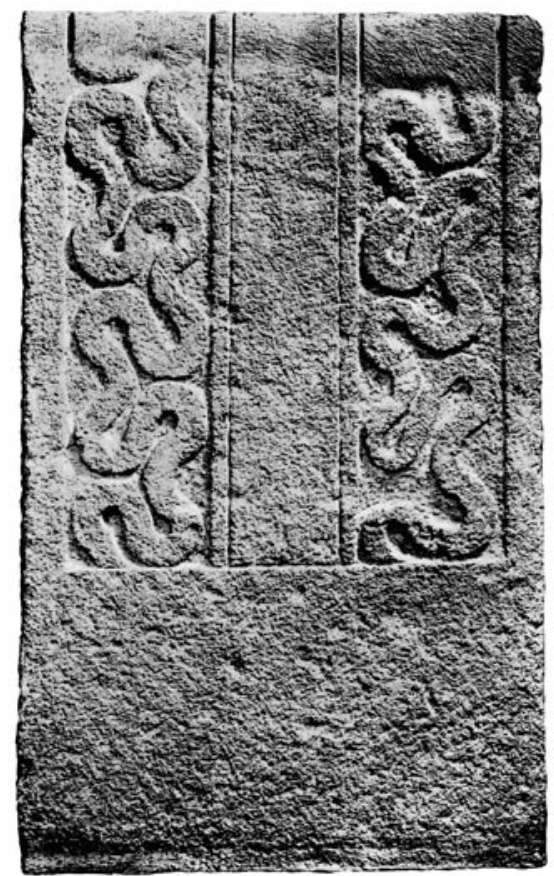

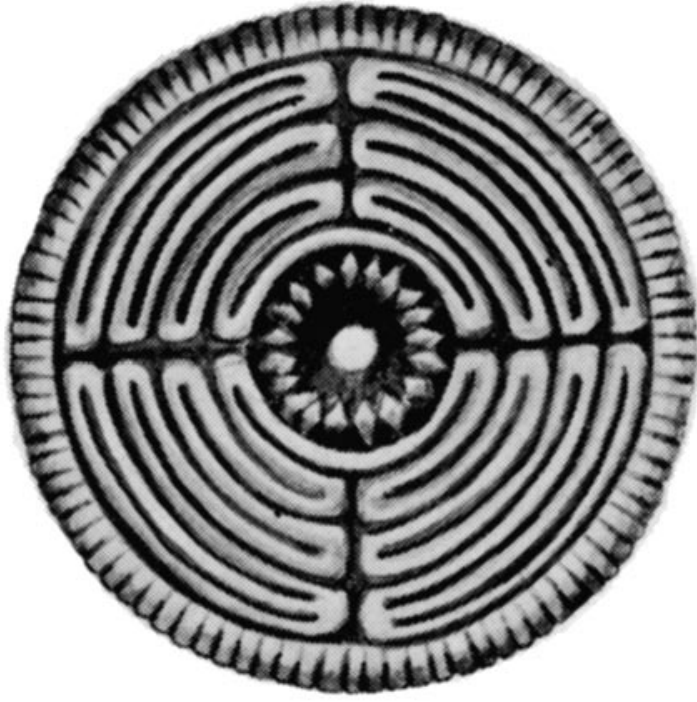

23 Goldblech aus Schachtgrab V in Mykene

22 Reliefstele über Schachtgrab II in Mykene

möglicherweise mehr als ein Tier, womit wir an die Tierreihe auf dem Elfenbeinkammgriff aus Schachtgrab III (Abb. 3) erinnert werden. Die Mähne des Löwen zur Linken ist im kleinteiligen Schraffurmuster wiedergegeben, und auf den Horror Vacui wird mit konventionellen gezackten Terrainmotiven an den Bildrändern, aber auch mit einem abstrakten Dreieck in einem Feldzwickel geantwortet. Bemerkenswert ist schließlich die breite Blattrankenzone im Zentrum dieses Siegelbildes, die in keiner inhaltlichen Relation zu den Figuren steht, sondern von einer ähnlich >unbedarften Steinstelen der Schachtgräber von Mykene (Abb. 7-10) zeugt.

Betrachten wir die heterogene Ornamentik der Schachtgräberzeit, so ist zum Vergleich auf eine Elfenbeinplakette mit S-Spirale aus dem MM IIB-zeitlichen `Quartier Mu< in Malia hinzuweisen ${ }^{73}$, die typologisch der Kamares-Ornamentik des protopalatialen Kreta ebenso nahesteht wie den wellenbandähnlichen Motiven auf dem jüngeren, schachtgräberzeitlichen Festland ${ }^{74}$. Eine noch deutlichere Verwandtschaft zwischen dem Ornamentdekor der kretischen Altpalastzeit und dem festländischen Formenspektrum der Schachtgräberzeit lässt der Akrobat auf der MM II-zeitlichen Schwertknaufmanschette aus Malia (Abb. 20) erkennen ${ }^{75}$. Sein detailliert geschilderter Schurz präsentiert ein T-förmiges Rapportmuster ${ }^{76}$, welches sich bei näherer Betrachtung als >flacher Mäander` erweist und eng vergleichbar dem Mäanderdekor auf Goldtreibarbeiten und weiteren Objekten aus den Schachtgräbern von Mykene ist ${ }^{77}$. Insbesondere die Rahmenzone einer Bildstele mit Wagenszenen aus Mykene (Abb. 21) zeigt denselben Flachmäander in einfacher Form ${ }^{78}$, und in stärker geschwungener Version schmückt ein Wellenmäander zwei vertikale Dekorfelder auf einer Stele über Schachtgrab II ${ }^{79}$ (Abb. 22). Wir werden somit auch das Ausgussstück des Straußenei-Rhytons

${ }^{73}$ Poursat (Anm. 43) 3-5 Taf. 1. Vgl. ferner Walberg 1986, $50 \mathrm{f}$.

${ }^{74}$ Vgl. den Spiraldekor auf dem goldenen Brustblech aus Schachtgrab V: Karo 1930, 122. 179 f. Nr. 625 Taf. 55.

${ }^{75}$ Chapouthier 1938, bes. 30-62. Zur Datierung s. O. Pelon, Peut-on dater les épées de Mallia?, BCH 106, 1982, 165-190, bes. 186 f. Abb. 27; O. Pelon, L'épée à l'acrobate et la chronologie maliote (II), BCH 107, 1983, 679-703; O. Pelon, L'acrobate de Malia et l'art de l'époque protopalatiale en Crète, in: Darcque - Poursat (Anm. 55) 35-40.

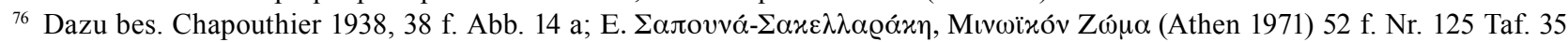
b; E. J. W. Barber, Prehistoric Textiles. The Development of Cloth in the Neolithic and Bronze Ages with Special Reference to the Aegean (Princeton 1991) 319 f. Abb. 15, 5.

77 s. u. a. Karo 1930, 270 Nr. 12. 16 Taf. 29; Nr. 320. 334 Taf. 60; Nr. 344-345 Taf. 61; Nr. 675 Taf. 63.

${ }_{78}$ Karo 1930, 170 Taf. 10 r. o.; Heurtley 1921-1923, 134 f. Nr. 8 a Abb. 30; Younger 1997, 236 Nr. 8 Taf. 91 d.

79 Karo 1930, 169 Nr. 1430 Taf. 8 1.; Heurtley 1921-1923, 129 f. Nr. 2 Abb. 28; Younger 1997, 235 Nr. 2 Taf. 88 c; 90 b. 
mit minoischen Delphinmotiven aus Schachtgrab V in Form eines Goldbleches mit vergleichbarem Dekor (Abb. 23) problemlos als minoischen Bestandteil dieses Importobjektes aus Kreta ansprechen können ${ }^{80}$. Diese Vergleiche machen deutlich, dass wir den >flachen Mäander keineswegs als >eurasischen` Motivimport nach Mykene zu verstehen brauchen ${ }^{81}$, sondern diese Ornamentik existierte offensichtlich bereits vor Beginn der Schachtgräberzeit zumindest in der minoischen Textilkunst, wo wir sie uns zweifellos in polychromer Form vorstellen dürfen. Dass so manches Ornament der Schachtgräberzeit Entsprechungen in der zeitgleichen und älteren Textilkunst Kretas besitzt, veranschaulichen etwa auch ineinander verschlungene Spiralvolutenmotive auf Reliefstelen in Mykene (Abb. 8 und 10), die als sich verzweigendes Kamares-Ornament auf dem Gürtel einer Männerfigur auf einem SM I-zeitlichen Freskofragment aus Knossos ebenso begegnen ${ }^{82}$ wie als von Kreta angeregtes Rapportmotiv auf älteren Deckenmalereien im Ägypten des Mittleren Reiches ${ }^{83}$.

Nicht nur die genannten Ornamentmotive können als genuin mittelminoisch verstanden werden, sondern eine Reihe von Siegeln und Siegelabdrücken unterschiedlicher Stätten auf Kreta bezeugt auch den indigenen Charakter mehrerer Varianten der Flecht- und Schlaufenbänder und deren Auftreten vor allem während MM II ${ }^{84}$. Dies bedeutet keineswegs, dass alle festländischen Motive dieses schachtgräberzeitlichen Ornamentstils als bloße Anregungen aus Kreta zu erklären sind; vielmehr sollte die Diskussion der Herkunft des \Schlaufen- und Schlingenstils` auf dem griechischen Festland unter stärkerer Differenzierung der Einzelmotive und auch mit Blick auf Kreta geführt werden. Dass die Ursprungsgeschichte mancher Ornamentmotive jedoch noch komplexer sein dürfte, veranschaulichen die Siegelabdrücke aus dem frühhelladischen Lerna ${ }^{85}$. Zwei in Vergesellschaftung mit einer SM III-zeitlichen Larnax geborgene Elfenbeinzylinder mit Wellenbanddekor und Kreisaugen aus dem ostkretischen Palaikastro hingegen bilden ein Unikum und müssen nicht zwingend mit dieser kretischen Stiltradition zusammenhängen ${ }^{86}$.

${ }^{80}$ Karo 1930, 125 Nr. 651 Taf. 141. 142; Foster (Anm. 31) 130-134 Abb. 88. Taf. 41. 42; J. A. Sakellarakis, The fashioning of ostrich-egg rhyta in the Creto-Mycenaean Aegean, in: D. A. Hardy u. a. (Hrsg.), Thera and the Aegean World III. Proceedings of the Third International Congress I (London 1990) 293 Abb. 33-39; 301. 305 f. Abb. 57.

${ }^{81}$ Penner 1998, bes. 163 f. Taf. 45, 2. Zu ähnlichen Ornamentmotiven im Balkanraum und anderen Regionen s. A. F. Harding, The Mycenaeans and Europe (London 1984) 191 Abb. 48, 2; David 2001, 54-57 Abb. 2. 3.

${ }^{82}$ Evans 1921, 201 Taf. 1 k; Evans 1935, 200 Abb. 110 A, k; W. S. Smith, Interconnections in the Ancient Near East. A Study of the Relationship between the Arts of Egypt, the Aegean, and Western Asia (New Haven - London 1965) 18 Abb. 33 c; E. $\Sigma \alpha \pi$ ovvó- $\Sigma \alpha x \varepsilon \lambda \lambda \alpha \varrho \alpha ́ x \eta$ (Anm. 76) 177 f. Abb. 75 o. Taf. D a (о.); M. A. S. Cameron, New restorations of Minoan frescoes from Knossos, BICS 17, 1970, 164 f.; M. A. S. Cameron, Theoretical interrelations among Theran, Cretan and Mainland frescoes, in: Ch. Doumas (Hrsg.), Thera and the Aegean World. Papers Presented at the Second International Scientific Congress, Santorini, Greece, I (London 1978) 579. 587 f. Abb. 4; M. A. S. Cameron, Diskussionsbeitrag, in: Ch. Doumas (Hrsg.), Thera and the Aegean World. Papers Presented at the Second International Scientific Congress, Santorini, Greece, II (London 1980) 315-317; D. Evely (Hrsg.), Fresco: A Passport into the Past. Minoan Crete through the Eyes of Mark Cameron (Athen 1999) 252-255 mit Farbabb.

${ }^{83}$ Barber (Anm. 76) 340-351. Vgl. weiters F. Blakolmer, Ikonographische Beobachtungen zu Textilkunst und Wandmalerei in der bronzezeitlichen Ägäis, ÖJh 63, 1994, Beibl. 1-28; M. C. Shaw, Anatomy and execution of complex Minoan textile

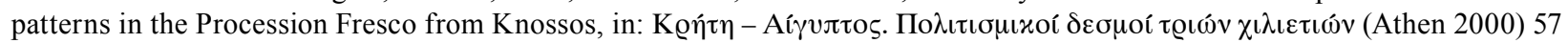
Abb. 5; M. C. Shaw, Grids and other drafting devices used in Minoan and other Aegean wall painting. A comparative analysis including Egypt, in: K. P. Foster - R. Laffineur (Hrsg.), Metron. Measuring the Aegean Bronze Age. Proceedings of the 9th International Aegean Conference, New Haven, Yale University, 18-21 April 2002, Aegaeum 24 (Liège - Austin 2003) 187 Taf. 44 f; M. Bietak - N. Marinatos - C. Palyvou, The maze tableau from Tell el Dab a, in: S. Sherratt (Hrsg.), Proceedings of the First International Symposium \The Wall Paintings of Thera<, 30 August - 4 September 1997, I (Athen 2000) 84-86 Abb. 7.

${ }^{84}$ s. z. B. Siegelabdrücke aus Knossos (CMS II 8 Nr. 21-22), ein Elfenbeinkonoid aus Tholos A in Platanos (CMS II 1 Nr. 313) und vor allem Abdrücke aus dem >Archivio di cretuleく in Phaistos (CMS II 5 Nr. 150-156. 159. 162-168). Dazu auch Pini 2004.

${ }^{85}$ s. M. Heath Wiencke in: CMS V 1, 28-32 Nr. 43-149; M. Heath Wiencke, Early Helladic clay sealings from the House of the Tiles at Lerna, Hesperia 27, 1958, 81-120; M. Heath Wiencke, The Lerna sealings, in: Die kretisch-mykenische Glyptik und ihre gegenwärtigen Probleme (Bonn - Bad Godesberg 1974) 149-172. Anregend ist die Betrachtungsweise der >Sealings` aus dem >House of the Tiles` als Indizien für anatolische Händler in Lerna von J. Weingarten, Another look at Lerna: An EH IIB trading post?, OxfJA 16, 1997, 147-166.

${ }^{86}$ R. C. Bosanquet - R. M. Dawkins, The Unpublished Objects from the Palaikastro Excavations 1902-1906, BSA Suppl. 1 (London 1923) 127 f. Abb. 110; J.-C. Poursat, Les ivoires mycéniens. Essai sur la formation d'un art mycénien, BÉFAR 230 (Paris 1977) 181 Taf. 11, 3. Zu einem Stück aus Tell Açana mit guter Entsprechung in Dekor und Gerätform s. Kull (Anm. 43) 68. 70 Abb. 15, 9. 
Da der mögliche Zusammenhang mehrerer Motiveigenarten der festländischen Bildwerke der Schachtgräberzeit mit kretischen Motiven aus FM III-MM II keine Synchronisierung zulässt ${ }^{87}$, legt dieses Phänomen nahe, dass nicht erst in MH III, sondern bereits in vorangegangenen Entwicklungsstadien des Mittelhelladikums auf dem Sektor der Bildkunst gewisse Kontakte mit Kreta bestanden. Betrachten wir die angeführten Analogien in der Siegelglyptik des protopalatialen Kreta unter dem Blickwinkel der Regionalstile, so fällt auf, dass Beispiele aus dem nördlichen Zentralkreta bei den Konvergenzen in der ikonographischen Gestaltung überwiegen, während das >Archivio di cretule in Phaistos zahlreiche Entsprechungen lediglich in der Ornamentsprache aufweist ${ }^{88}$. Zwar muss diese Verteilung keineswegs als repräsentativ gelten, jedoch ist bemerkenswert, dass eine Reihe von Siegelabdrücken mit figürlichen Motiven aus dem MM IIB-zeitlichen >Archivio in Phaistos bereits entwickeltere Tendenzen der Anfänge naturalistischer Bildsprache erkennen lässt, was in Malia zu dieser Zeit wiederum in der Ikonographie anderer Kunstgattungen, der Reliefkeramik und der Goldtreibarbeit, der Fall ist ${ }^{89}$, nicht jedoch in der Siegelglyptik. Dies könnte nahelegen, dass insbesondere Siegelbilder nordkretischer Werkstätten gewisse Anregungen auf die Zentren des mittelhelladischen Festlandes ausübten. Unklar bleibt auch, welche Rolle Ägina bei diesem Vermittlungsprozess zukam $^{90}$, doch scheinen diesbezügliche Mutmaßungen beim heutigen Wissensstand zweifellos sehr gewagt. Für unsere Frage nach der Existenz einer mittelhelladischen Figuralkunst ist weniger die konkrete Verbindung mit Kreta von Interesse als vielmehr das chronologische Phänomen, dass diese minoischen Stiltendenzen eine weit zurückreichende Tradition besitzen und in MM III, d. h. etwa zeitgleich mit dem Beginn der Schachtgräberzeit auf dem helladischen Festland, auf Kreta bereits ausgelaufen waren und schwerlich erst jetzt wirksam werden konnten. Sollten die hier festgestellten Zusammenhänge Gültigkeit besitzen, so muss die Entstehung mancher autochthon-festländischer Kunsttendenzen in Bildwerken der Schachtgräberzeit in früheren Phasen des Mittelhelladikums anzusetzen sein.

\section{Zeugnisse einer mittelhelladischen Bildtradition?}

Die festländische Bildkunst der Schachtgräberzeit wurde zweifellos aus mehreren Richtungen und Traditionen gespeist. Deutlich ist das Einwirken zeitgleicher Bildwerke aus dem neopalatialen Kreta, und auch die nach wie vor unkonkreten, am ehesten ägyptischen Vorbilder für die Motive des kämpfenden Kriegers oder Herrschers im Streitwagen dürften erst in SH I nach Mykene gelangt sein. Auf einer anderen Zeitebene, und zwar deutlich im Mittelhelladikum verhaftet, könnten hingegen manche `Minoismen` anzusiedeln sein, die einen vitalen Bestandteil des festländischen Elementes bilden. Wie zuvor gezeigt wurde, dürfte es sich hierbei um genuin kretische Motive und Darstellungskonventionen handeln, die den Entwicklungsperioden vor MM III angehören. Wann und wie auch immer sich helladische Künstler diese Anregungen aus dem altpalastzeitlichen oder noch älteren Kreta geholt haben mögen - dies geschah jedenfalls vor dem deutlichen Einsetzen des massiven Importes kretischer Preziosen mit Bilddekor, den wir erst während der Schachtgräberzeit annehmen können. Wie stark ausschnitthaft die Primärevidenz an erhaltenen autochthonen sowie importierten Bildobjekten generell ist, verdeutlicht allein schon der Umstand, dass uns die kretischen Vorlagen für >minoisierendeく Bildmotive wie auf den Stelen des Gräberrundes B (Abb. 7) in keinem einzigen Beispiel erhalten sind und dennoch vorausgesetzt werden müssen ${ }^{91}$. Es spricht somit einiges dafür, dass wir

87 s. bes. Warren - Hankey (Anm. 54) 46-65. 67-69. 96-98.

${ }^{88} \mathrm{Zu}$ den Landschaftsstilen in der altpalatialen Siegelglyptik Kretas s. E. Thomas, Minoisches Siegelwesen, in: Im Labyrinth des Minos. Kreta - die erste europäische Hochkultur, Ausstellungskatalog Badisches Landesmuseum Karlsruhe (München 2000) 145; Galanakis 2005, 41-48; E. Thomas, Bilder als Medien. Das Siegelbild als Mittel der Kommunikation in der minoischen Palastzeit, in: Medien in der Antike. Kommunikative Qualität und normative Wirkung (Köln 2003) $205-206$.

89 J.-C. Poursat, Reliefs d'applique moulés, in: B. Detournay - J.-C. Poursat - F. Vandenabeele, Fouilles exécutées à Mallia. Le quartier Mu II, ÉtCrét 26 (Paris 1980) 116-130. Zum Akrobaten auf der Griffmanschette s. o. Anm. 75.

90 Dazu S. Hiller, Minoan and Minoanizing pottery on Aegina, in: C. Zerner - P. Zerner - J. Winder (Hrsg.), Proceedings of the International Conference Wace and Blegen. Pottery as Evidence for Trade in the Aegean Bronze Age 1939-1989 (Amsterdam 1993) 197-199; W. Gauß, Minos auf Ägina - Beobachtungen zu den Beziehungen Äginas zu Kreta, in: E. Czerny u. a. (Hrsg.), Timelines. Studies in Honour of Manfred Bietak II, Orientalia Lovaniensia Analecta 149 (Leuven 2006 ) $437-448$. s. ferner u. (Anm. 101).

91 s. Pini 1985, 156 f. 
in den erörterten Reliefwerken der Schachtgräberzeit tatsächlich die Reste einer eigenen Figuralkunst mit weiter zurückreichenden Wurzeln im früheren Mittelhelladikum fassen.

Wie haben wir uns diese festländische Bildwelt des Mittelhelladikums konkret vorzustellen? Kein schachtgräberzeitliches Beispiel zeigt diese helladische Bildkunst noch in ihrer reinen, ungebrochenen Ausformung; dies könnte am ehesten noch beim Goldblech mit Tierfries aus Schachtgrab III (Abb. 3) sowie dem Silberbecher mit Löwenjagd aus Schachtgrab Delta (Abb. 1) der Fall sein. Mit Ausnahme der Wagenkampfszenen lässt die vorliegende helladische Bildwelt keine eigenständigen Bildthemen, sondern bestenfalls gewisse Präferenzen erkennen, treffen wir doch Tierüberfall und Löwen auch im kretischen Motivspektrum $a^{92}$. Die herausgearbeiteten Stilmerkmale dieser schachtgräberzeitlichen Reliefbilder sind nicht bloß durch eine Tendenz zu Schlichtheit und Schematismus gekennzeichnet, sondern auch durch Ornamentalisierung und Geometrisierung. Die helladischen Figurenszenen der Schachtgräberzeit zeugen jedoch auch von einer nur wenig standardisierten Bildsprache. Die Spezifika der Kompositionsweise lassen eine deutlich geringere Komplexität als die imitierten minoischen Prototypen erkennen; auch die Wagenkampfszenen in nahöstlicher Motivtradition werden wir schwerlich bereits vor der Schachtgräberzeit ansetzen dürfen. Zwar erscheint es plausibel, dass Toreuten die treibende Kraft der festländischen Kunst während der Schachtgräberzeit bildeten ${ }^{93}$, doch könnten die tatsächlichen Ursprünge dieses Reliefstils eher im Handwerk der Schnitzerei liegen. Nicht zufällig wurde die Reliefierung der Steinstelen in zwei Ebenen häufig als $>$ Holzschnitzstik charakterisiert ${ }^{94}$. Die Flächenorientiertheit in der Reliefgestaltung und manche graphische Elemente legen eine bescheidene mittelhelladische Bildkunst in Malerei, in Schnitzarbeiten oder auch auf Textilien nahe.

Wie eingangs erwähnt, lassen uns nahezu alle erhaltenen Bildmedien bei der Definition einer mittelhelladischen Figuralkunst im Stich. Die schematischen gemalten Figuren auf Gefäßfragmenten ${ }^{95}$ sind motivisch und stilistisch so heterogen und individuell, dass sie schwerlich einer konsistenten Bildkunst auf breiterer Basis angehören können. Der von W. A. Heurtley angestellte Vergleich von Ikonographie und Stil der Stelenbilder von Mykene mit der mittelhelladischen und mittelkykladischen Keramik ${ }^{96}$ erscheint zu optimistisch ${ }^{97}$ und geht über eine >geometrische Tradition< der Ornamentik, horizontale und vertikale Gliederung etc. kaum hinaus; mit der überaus bildfreudigen Keramikmalerei des späten Mittelkykladikums hat diese festländische Kunstrichtung kaum etwas gemeinsam ${ }^{98}$. Es sei daran erinnert, dass selbst im altpalastzeitlichen Kreta der mittelminoische Keramikdekor weitgehend anikonisch bleibt; sieht man von wenigen Ausnahmen schematischer Figuren auf Gefäßen im Kamares-Stil ab, so beschränkt sich die protopalatiale Bildwelt Kretas auf die Siegelglyptik und entwickeltere Beispiele in den meist reliefierten Preziosen. Umso bemerkenswerter sind die Darstellungen von Booten mit Ruderern bzw. Kriegern in der mittelhelladischen Keramik von Kolonna auf Ägina ${ }^{99}$. Auch Beispiele der Siegelglyptik auf dem mittelhelladischen Festland ergeben für unsere Fragestellung bislang keinerlei Ergebnisse ${ }^{100}$; lediglich Neufunde aus Kolonna dürften

${ }_{92}$ Pini 1985. Zur gesammelten Bildevidenz für Löwenmotive s. Vanschoonwinkel (Anm. 68) 362 f. 398-400.

${ }^{93}$ s. Vermeule 1975, 10 f. 38.

${ }_{94}$ Zur Ableitung des Reliefstils der Steinstelen aus dem geschnitzten Holzbild s. Hood 1978, 99; Younger 1997, 233. Damit vergleichbar sind auf Kreta lediglich die Fayencetafeln des \Stadt-Mosaiks` aus Knossos. So bereits Müller 1915, 268. Ähnlich auch Heurtley 1921-1923, 137.

${ }^{95}$ s. o. Anm. 5.

${ }^{96}$ Heurtley 1921-1923, 140-144. s. auch O. Frödin - A. W. Persson, Asine. Results of the Swedish Excavations $1922-1930$ (Stockholm 1938) 257.

${ }^{97}$ s. auch die Kritik von Evans (Anm. 19:1929) 57.

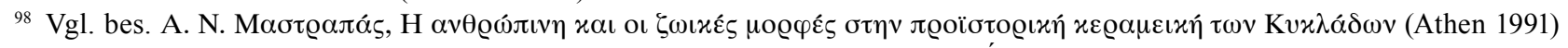
181-191; G. Sakellarakis, Le thème du pêcheur dans l'art Préhistorique de l'Égée, AAA 7, 1974, 371-373 Abb. 1. 2; Ch. G.

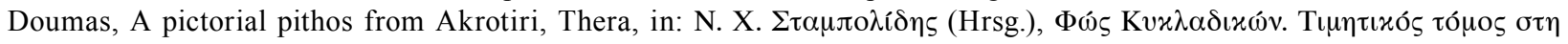

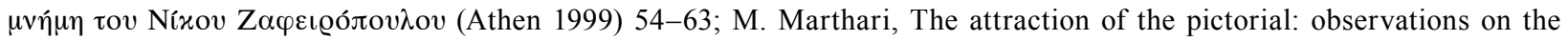
relationship of Theran pottery and Theran fresco iconography, in: S. Sherratt (Hrsg.), Proceedings of the First International Symposium >The Wall Paintings of Thera<, 30 August - 4 September 1997, II (Athen 2000) 873-889.

99 s. o. Anm. 5 sowie G. Welter, Aigina (Berlin 1938) 19 Abb. 22; S. Hiller, Fisch oder Schiff. Zu einem bemalten mittelbronzezeitlichen Gefäßfragment aus Ägina, Pantheon 30, 1972, 439-446.

${ }^{100}$ Dazu J. G. Younger, Seals? from Middle Helladic Greece, Hydra 8, 1991, 35-54; I. Pini, Minoische und helladische Tonsiegel, in: Aux origines de l'hellénisme. La Crète et la Grèce, Festschrift Henri van Effenterre (Paris 1984) 75; I. Pini, Minoische 
hier weiteren Aufschluss versprechen ${ }^{101}$. Es sei jedoch davor gewarnt, die Existenz einer Figuralkunst an die Verwendung von Siegeln als Bildträger zu knüpfen.

Die Frage nach den gesellschaftlichen Voraussetzungen und Erfordernissen für eine Ikonographie an den Zentren des mittelhelladischen Festlandes kann zur Lösung des Problems ebenfalls kaum etwas beitragen. Der verhältnismäßig geringe Stellenwert von >community interests $\measuredangle$ in den nur wenig kompetitiven Gesellschaften des mittelhelladischen Griechenland lässt kaum autochthone Prestigeobjekte mit Bildkunst erwar$\operatorname{ten}^{102}$. Lediglich die in der zu rekonstruierenden Bildwelt stark ausgebildete Polarisierung der Geschlechteridentitäten entspricht unseren traditionellen Erwartungen gegenüber frühen Kriegergesellschaften. Vordergründig spricht nichts dafür, dass im Mittelhelladikum eine `Sprache der Bilder Bestandteil der kulturellen Ideologie und der >sozialen Strategien` dieser Zeit war, doch wissen wir viel zu wenig über die Möglichkeiten und Grenzen der Nutzbarkeit von Ikonographie, als dass sich daraus Fixpunkte für die vorliegende Diskussion erarbeiten ließen ${ }^{103}$. Lassen wir Aspekte des Stils und der ikonographischen Konventionen beiseite, so muss in den festländischen Dorfgemeinschaften wohl lediglich die Bereitschaft zur Darstellung von Raubtieren und von Kriegern gegeben gewesen sein. Bei Betrachtung der frühkykladischen Steinplatten aus Korphi t'Aroniou auf Naxos mit ihren mehrfigurigen Bildszenen schematischer Jäger, Viehzüchter, Krieger und Seefahrer gewinnen wir nicht nur eine gewisse Vorstellung der Komplexität eines grundsätzlich vergleichbaren ikonographischen Spektrums ${ }^{104}$. Dieser Vergleich führt uns auch vor Augen, dass Bildthemen wie diese in mehrfiguriger Ikonographie in der Altägäis keineswegs nur in entwickelteren, streng hierarchisch strukturierten Gesellschaften, sondern auch in der protourbanen Welt ihren Platz finden konnten.

\section{Liegen hier die Anfänge einer >mykenischen Kunst` vor?}

Können wir in der hier herausgearbeiteten autochthon-helladischen Bildsprache mit Wurzeln im früheren Mittelhelladikum die Anfänge einer >mykenischen Kunst erkennen, wie dies in der Forschung für manche festländische Eigenarten während der Schachtgräberzeit oft postuliert wurde ${ }^{105}$ ? In der Schachtgräberzeit zwischen >minoisierend und einem konstruierten prospektiven >mykenisch zu unterscheiden, ist nicht nur methodisch problematisch, sondern $\mathrm{m}$. E. nicht einmal zulässig. Was wir herausfiltern konnten, ist ein >mittelhelladischer Anteil in der Gestaltung mancher Bilddenkmäler, welcher mit der uns vertrauten Bildkunst des zeitgleichen minoischen Kreta in deutlichem Kontrast steht. Der hybride, experimentelle und oft ephemere Charakter der festländischen Bildwerke dieser Zeit lässt zwar eine Bildsprache im Wandel erkennen, erlaubt es m. E. jedoch nicht, hier die Anfänge einer konsistenten neuen Bildkunst auf dem griechischen Festland zu fixieren. Dies dürfte sogar noch für die ikonographischen Beispiele der darauffolgenden Entwicklungsphasen SH IIB-IIIA1 Gültigkeit besitzen; sind doch auch komplexere Bildwerke wie die >Oktopustasse ${ }^{106}$ und der Silberkelch mit jagenden Hunden aus Dendra ${ }^{107}$ oder die Elfenbeinpyxis mit

Siegel außerhalb Kretas, in: R. Hägg - N. Marinatos (Hrsg.), The Minoan Thalassocracy. Myth and Reality, Proceedings of the Third International Symposium at the Swedish Institute in Athens, 31 May - 5 June, 1982 (Stockholm 1984) 123-131; I. Pini, Some considerations on the use of seals for administrative purposes in Mycenaean Greece, SMEA 28, $1990,108$.

101 s. W. Gauss, Aegina-Kolonna in the Middle Bronze Age, in: A. Philippa-Touchais u. a. (Hrsg.), MESOHELLADIKA. The Greek Mainland in the Middle Bronze Age, International Conference, Athens, 8-12 March 2006, BCH Suppl. (in Druck). Herrn Walter Gauß bin ich sehr dankbar, dass er mir Einblick in Neufunde gewährte.

102 Rutter 1993, 780. 790.

${ }^{103}$ Sehr anregend zu grundsätzlichen Fragen der >Bildwürdigkeit`mancher Themen in der frühägäischen Bildwelt ist Ch. Gates, Why are there no scenes of warfare in Minoan art?, in: Laffineur (Anm. 48) 277-283.

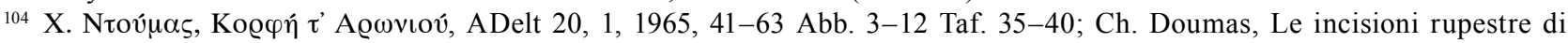
Nasso, nelle Cicladi, Bollettino del Centro Camuno di Studi Preistorici 3, 1967, 111-132; Ch. Doumas, Silent Witnesses. Early Cycladic Art of the Third Millennium BC (New York 2002) 40 Abb. 47; L. Marangou (Hrsg.), Cycladic Culture. Naxos in the 3rd Millennium BC (Athen 1990) 82 Nr. 80-81; 89 Nr. 88; 116 Nr. 112; C. Broodbank, An Island Archaeology of the Early Cyclades (Cambridge 2000) 97-99 Abb. 23.

105 s. o. Anm. 6 und 20.

106 A. W. Persson, The Royal Tombs at Dendra near Midea (Lund u. a. 1931) 43-48 Frontispiz. Taf. IX-XI; Laffineur (Anm. 24) 112 f. Nr. 81 Abb. 36. 37; Hurwit 1979, bes. 416-426.

${ }_{107}$ Persson (Anm. 106) 51-54 Abb. 30 Taf. 17; Smith (Anm. 82) 72 Abb. 98; B. Kaiser, Untersuchungen zum minoischen Relief (Bonn 1976) 163 a. b; Davis 1977, 282 f. Nr. 118 Abb. 229. 


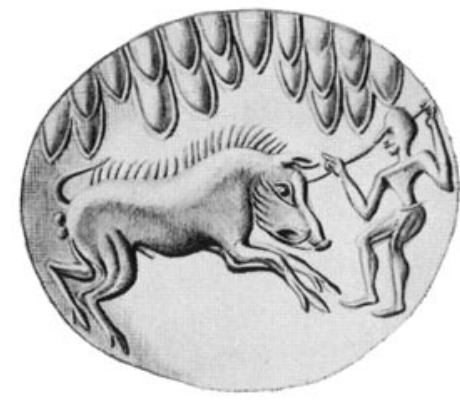

24 Siegel aus Vapheio

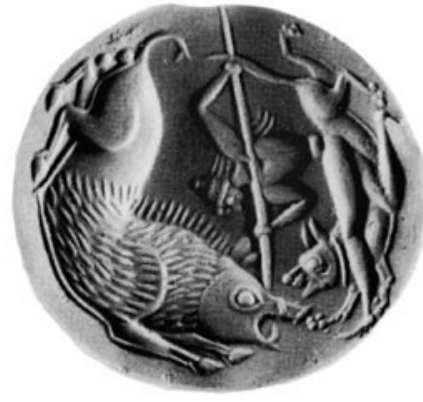

25 Siegel aus Pylos (Vagenas)

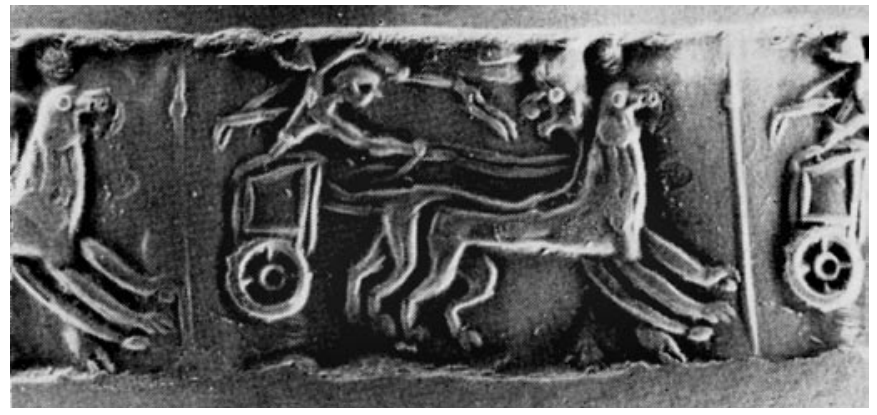

26 Siegel aus Kazarma

Greifenjagd aus Athen ${ }^{108}$ geprägt von künstlerischer Distanz, Inkonsistenz und so manchem Missverstehen altkretischer Darstellungskonventionen. Die hybriden, experimentellen und stets individuellen >Versuche< der Schachtgräberzeit stellen zweifellos nicht das Fundament für die spätere Bildkunst des palastzeitlichen Festlandes während SH IIIA2-IIIB2 dar. Eine Kontinuität besteht hier m. E. lediglich in der Bereitschaft zum Import sowie zum mehr oder weniger kreativen, freien Umgang mit neopalatial-kretischen (Erb-)Stücken. Weder die schachtgräberzeitlichen ikonographischen Ausformungen des Kriegers im Pferdegespann (Abb. 9-11) oder des Löwen im >Absprunggalopp (Abb. 1) noch die genannten stilistischen Eigenheiten finden eine wesentliche Fortsetzung oder Weiterführung in SH IIA-IIIA1 oder in der nachfolgenden mykenischen Palastzeit.

Überaus spärlich sind die vereinzelten Nachläufer dieser schachtgräberzeitlichen Formensprache in späterer Zeit. Zwar lassen sich im festländischen >Palaststik möglicherweise teils noch ältere Dekorschemata und Gestaltungselemente erkennen ${ }^{109}$, doch kann dieser Anteil kaum als substanziell verstanden werden, und dies gilt ebenso für die Ikonographie. So zeigt etwa ein Lentoidsiegel mit Keilerjagd aus der SH IIAzeitlichen Tholos von Vapheio (Abb. 24) einen angreifenden Jäger in leichter Hocke und mit unorganisch abstehenden Armen ${ }^{110}$, vergleichbar manchen Figuren auf Bildstelen in Mykene (Abb. 9. 10. 12). Ähnlich wie im Falle eines Kriegers in einem Stelenbild (Abb. 9) wird hier die Waffe nicht in den Händen gehalten,

108 T. L. Shear, The campaign of 1939, Hesperia 9, 1940, 283-288 Abb. 27-29; S. A. Immerwahr, The Neolithic and Bronze Ages, Agora 13 (Princeton 1971) 106. 166 (I-16) Taf. 32.

${ }^{109}$ K. Kalogeropoulos, Die frühmykenischen Grabfunde von Analipsis. Mit einem Beitrag zu den palatialen Amphoren des griechischen Festlandes (Athen 1998) bes. 159-174; K. Kalogeropoulos, Decorative schemes as an indicator of artistic relations between early Mycenaean Greece and the eastern Mediterranean, in: R. Laffineur - E. Greco (Hrsg.), EMPORIA. Aegeans in the Central and Eastern Mediterranean. Proceedings of the 10th International Aegean Conference, Athens, Italian School of Archaeology, 14-18 April 2004, Aegaeum 25, 1 (Liège - Austin 2005) 393-402; O. T. P. K. Dickinson, Late Helladic IIA and IIB. Some evidence from Korakou, BSA 67, 1972, 103-112; Niemeier (Anm. 3) 116 f.

${ }^{110}$ CMS I Nr. 227. Dazu nun F. Blakolmer, Zum Lentoidsiegel mit Keilerjagd aus dem frühmykenischen Tholosgrab von Vapheio, in: Ch. Franak u. a. (Hrsg.), Thiasos. Festschrift Erich Pochmarski (Wien 2008) 89-100. 
sondern erscheint abstrakt beigefügt - doch bleibt dies ein Einzelfall in der frühmykenischen Bildkunst des SH II. Dass sich die spätere Ikonographie des Streitwagens von den Motiven der Schachtgräberstelen grundsätzlich unterscheidet, wurde bereits erwähnt. Umso bemerkenswerter ist daher die Darstellungsweise des Wagens auf dem zuvor genannten Rollsiegel aus dem SH IIA-Tholosgrab von Kazarma ${ }^{111}$ (Abb. 26), welche den Wagenkästen auf den Stelenbildern in Mykene (Abb. 9-11) verblüffend nahe kommt. Auch eklatante Mängel in Maßstabstreue und Proportionierung begegnen gelegentlich noch in jüngeren Bildwerken, so etwa auf einem Siegel mit einem kleinformatigen Jäger mit Hund und einem übergroßen Keiler aus der SH I-IIIA-zeitlichen Tholos V in Pylos (Grundstück Vagenas) ${ }^{112}$ (Abb. 25). All diesen Beispielen ist gemeinsam, dass sich Ikonographie und Stil sonst stark an kretischen Traditionen orientieren, sodass wir hier lediglich noch gewisse Restelemente der festländischen Bildsprache mit möglicherweise älteren Wurzeln antreffen. Trotz der punktuellen kunstgeschichtlichen Nachläufer besaß dieser festländische Stil mittelhelladischen Ursprungs eines sicherlich nicht: Zukunft. Die Zukunft der Bildkunst im mykenischen Griechenland liegt jedenfalls in den ikonographischen Motiven und Stilmöglichkeiten des neopalatialen Kreta selbst und geht möglicherweise auf Importstücke zurück, welche bereits den schachtgräberzeitlichen Künstlern als Vorlage gedient haben könnten. Es mag zwar paradox klingen, dürfte jedoch den Kern des Problems treffen: Um wirklich >mykenisch zu sein, fehlt den Stelenbildern und anderen festländischen Bildwerken der Schachtgräberzeit noch sehr viel Minoisches.

\section{Schlussfolgerungen}

Der Aussage von J. Hurwit, »... there is little evidence for a coherent Middle Helladic artistic tradition ... «113, kann zwar selbst heute nicht widersprochen werden, doch dürften sich zumindest die Indizien dafür verdichtet haben, dass die neopalatial-kretische Kunst auf dem schachtgräberzeitlichen Festland keineswegs auf ein Nichts stieß, sondern hier bereits zuvor eine bescheidene autochthone Formensprache und figurale Bildkunst mit weiter zurückreichenden Wurzeln bestanden hatten. Nicht viel lässt sich über diese rekonstruierte mittelhelladische Bildkunst aussagen. Ihre Eigenheiten erscheinen jedenfalls zu spezifisch und zu homogen, um als beliebig oder individuell abgetan zu werden, und mit einer gewissen Tradition sollte gerechnet werden. Die Bildwelt des Mittelhelladikums reichte wohl über isolierte oder einige wenige interagierende Einzelfiguren in schematischer Form nicht hinaus, wobei dem Ornament als Rahmung sowie auch im Motivaufbau eine wesentliche Rolle zukam. Die bescheidene Komplexität der Ikonographie dürfte in etwa jener des mittelminoischen Kreta entsprochen haben, und zwar vor dem Einsetzen des `kretischen Naturalismus々 gegen Ende von MM II.

Zwei Punkte seien abschließend hervorgehoben. Wenn unsere Vorstellungen einer indoeuropäischen Einwanderung auf das griechische Festland in der späten Frühbronzezeit zutreffen, so ist das, was wir in den hier diskutierten Bildwerken an Mittelhelladischem fassen, die erste 'griechische Kunst‘. Folgenschwerer für unsere Beurteilung der Bilddenkmäler der Schachtgräberzeit bzw. aus frühmykenischen Kontexten scheint jedoch ein weiterer Umstand: Wenn die hier präsentierten Bildwerke typisch für das helladische Kunstschaffen während der Schachtgräberzeit sind, so sollten wir zahlreiche vermeintlich >frühmykenische< Preziosen wie etwa den silbernen \Krieger-Krater< und das `Belagerungsrhyton〈 aus Schachtgrab IV sowie den Großteil der Steinsiegel, Goldringe und auch Metalleinlagedolche mit ihrer vielseitigen Ikonographie, unter anderem mit Bildszenen von Jagd und Kampf, in ihrem ursprünglich kretisch-minoischen Kontext verstehen.

\footnotetext{
111 s. o. Anm. 53.

112 CMS I Nr. 294; Krzyszkowska 2005, 251 Abb. 479.

113 Hurwit 1979, 424.
} 


\section{Katalog der wichtigsten autochthon-helladischen Bildwerke der Schachtgräberzeit}

1. Silberbecher mit Löwenjagd aus Schachtgrab Delta in Mykene (Abb. 1)

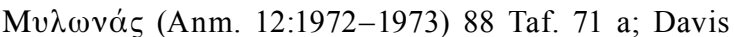
1977, 136 f. Nr. 30 Abb. 105; $\Xi \varepsilon v \alpha ́ x \eta-\Sigma \alpha x \varepsilon \lambda \lambda \alpha \varrho i ́ o v$ (Anm. 39) 61; N. R. Thomas, The war animal: three days in the life of the Mycenaean lion, in: Laffineur (Anm. 48) 300-302 Taf. 63, Nr. 1. 13; Thomas (Anm. 68) 174 Abb. 9. 18; 194.

2. Goldplaketten einer Holzschatulle aus Schachtgrab $\mathrm{V}$ in Mykene (Abb. 2)

C. Schuchhardt, Schliemann's Ausgrabungen in Troja, Tiryns, Mykenä, Orchomenos, Ithaka im Lichte der heutigen Wissenschaft (Leipzig 1891) 298-302 Abb. 274-277; Müller 1915, 294-297 Abb. 16; Karo 1930, 143 f. Nr. 808-811 Taf. 143. 144; Vermeule 1975, 24 f. Abb. 26. 27; Marinatos - Hirmer 1973, Taf. 220. 221.

3. Goldblech eines Elfenbeinkammes mit Tierfries und >minoisierendem`Ornamentband mit Wellenmäander und Rosetten auf der Rückseite aus Schachtgrab III in Mykene (Abb. 3)

Tierfries: Karo 1930, 58 Nr. 109 Taf. 32 u.; 33 о.; Г. A.

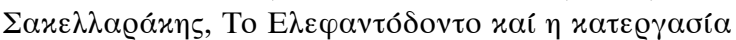

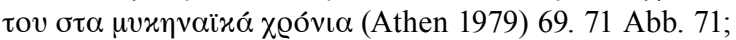
Velsink 2003, 2 (C 1) Abb. 2; S. 18-20. - Wellenmäander: Velsink 2003, 2 (C 1) Abb. 1; S. 18 f.

4. Goldener Nadelkopf mit Löwen und Greifen aus Schachtgrab IV in Mykene (Abb. 4)

Karo 1930, 78 Nr. 274 Taf. 32 o.; I. Kilian-Dirlmeier, Nadeln der frühhelladischen bis archaischen Zeit von der Peloponnes, PBF 13, 8 (München 1984) 44 f. Nr. 94 Taf. 3, 94; K. Demakopoulou (Hrsg.), Troja, Mykene, Tiryns, Orchomenos. Heinrich Schliemann zum 100. Todestag, Ausstellungskatalog (Athen 1990) 298 Nr. 247 mit Farbtaf.

5. Goldblech mit Männerprozession (?) aus Tholos 1 in Peristeria, SH IIA (Abb. 5)

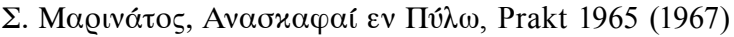
113 f. Taf. 128 c; S. Marinatos, Problemi archeologici e filologici di Pilo, SMEA 3, 1967, 11 Abb. 15; R. Hägg, On the nature of Minoan influence in early Mycenaean Messenia, OpAth 14, 1982, 32 f. Abb. 11; Y. G. Lolos, The Capital of Nestor and its Environs. Sandy Pylos (Athen 1998) 71 Abb. 35. - Zur Datierung der Tholos 1 s. M. J. Boyd, Middle Helladic and Early Mycenaean Mortuary Practices in the Southern and Western Peloponnese, BAR IntSer 1009 (Oxford 2002) 169. 172.

6. Goldblech mit gelagertem Greif aus Thorikos, SH I Spät - SH IIA (Abb. 6)

J. und B. Servais-Soyez, La tholos >oblongue (tombe IV) et le tumulus (tombe V) sur le Vélatouri, in: Thorikos VIII, 1952/1976 (Gent 1984) 47-50 Abb. 26, 6 a. b.
7. Reliefstele über Schachtgrab Gamma in Mykene (Abb. 7)

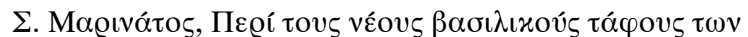

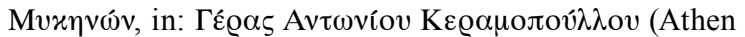
1953) 66-78 Abb. 2 Taf. 8. 9; Marinatos 1968, 175-177

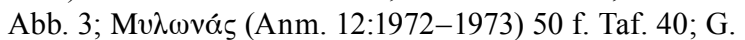
Graziadio, The process of social stratification at Mycenae in the Shaft Grave period: a comparative examination of the Evidence, AJA 95, 1991, 411; Younger 1997, 238 Nr. 14 Taf. 89 d.

8. Reliefstele aus Gräberrund A in Mykene (〉PferdeStele`) (Abb. 8)

Heurtley 1921-1923, 132 f. Taf. 21 u.; Younger 1997, 236 Nr. 6 Taf. 91 b.

9. Reliefstele über Schachtgrab V in Mykene (Abb. 9) Heurtley 1921-1923, 132 Taf. 20 o.; Mylonas 1951, 136-138 Abb. 2; Marinatos - Hirmer 1973, Taf. 169; Younger 1997, 236 Nr. 5 Taf. 91 a.

10. Reliefstele über Schachtgrab V in Mykene (Abb. 10) Heurtley 1921-1923, 131 Taf. 20 u.; Mylonas 1951, 137 f. Abb. 3; Younger 1997, 235 Nr. 4 Taf. 90 d.

11. Reliefstele über Schachtgrab V in Mykene (>plastische Stele $<$ ) (Abb. 11)

Müller 1915, 286-288 Abb. 13; Heurtley 1921-1923, 128 f. 143 Taf. 19; Mylonas 1951, 141-147 Abb. 6; Hood 1978, 99 f. Abb. 81; Marinatos - Hirmer 1973, Taf. 168; Younger 1997, 235 Nr. 1 Taf. 90.

12. Fragment einer Reliefstele aus Gräberrund $A$ in Mykene (Abb. 12)

Heurtley 1921-1923, 136 Nr. 10 a Abb. 31; K. Demakopoulou (Hrsg.), Troja, Mykene, Tiryns, Orchomenos. Heinrich Schliemann zum 100. Todestag, Ausstellungskatalog (Athen 1990) 317 Abb. 275; Younger 1997, 237 Nr. X Taf. 92 b; E. Rystedt, Iconographical notes on two stele fragments from Grave Circle A at Mycenae, in: P. M. Fischer (Hrsg.), Contributions to the Archaeology and History of the Bronze and Iron Ages in the Eastern Mediterranean. Studies in Honour of Paul Åström, SoSchrÖAI 39 (Wien 2001) 113 f. Abb. 1. 2. 


\section{Abgekürzt zitierte Literatur}

Zusätzlich zu den unter <http://www.oeai.at/publik/autoren.html $>$ und $<$ http://www.dainst.org/index_141_ de.html> angegebenen Abkürzungen werden hier folgende verwendet:

Blakolmer 1997

Chapouthier 1938

Crouwel 1982

David 2001

Davis 1977

Dickinson 1977

Evans 1921

Evans 1935

Galanakis 2005

Heurtley 1921-1923

Hood 1978

Hurwit 1979

Karo 1930

Krzyszkowska 2005

Marinatos 1968

Marinatos - Hirmer 1973

Müller 1915

Mylonas 1951

Penner 1998

Pini 1985

Pini 2004

Rutter 1993

Velsink 2003

Vermeule 1975

Walberg 1986

Wingerath 1995

Younger 1997
F. Blakolmer, Minoan wall-painting: The transformation of a craft into an art form, in: R. Laffineur - Ph. P. Betancourt (Hrsg.), TEXNH. Craftsmen, Craftswomen and Craftsmanship in the Aegean Bronze Age. Proceedings of the 6th International Aegean Conference, Philadelphia, Temple University, 18-21 April 1996, Aegaeum 16, 1 (Liège - Austin 1997) 95-105.

F. Chapouthier, Deux épées d'apparat découvertes en 1936 au palais de Mallia, ÉtCrét 5 (Paris 1938).

J. L. Crouwel, Chariots and Other Means of Land Transport in Bronze Age Greece (Amsterdam 1981).

W. David, Zu den Beziehungen zwischen Donau-Karpatenraum, osteuropäischen Steppengebieten und ägäisch-anatolischem Raum zur Zeit der mykenischen Schachtgräber unter Berücksichtigung neuerer Funde aus Südbayern, Anodos 1, 2001, 51-80.

E. Davis, The Vapheio Cups and Aegean Gold and Silver Ware (New York - London 1977).

O. T. P. K. Dickinson, The Origins of Mycenaean Civilization, SIMA XLIX (Göteborg 1977).

A. Evans, The Palace of Minos at Knossos I (London 1921).

A. Evans, The Palace of Minos at Knossos IV (London 1935).

K. Galanakis, Minoan Glyptics: Typology, Deposits and Iconography. From the Early Minoan Period to the Late Minoan IB Destruction in Crete, BAR IntSer 1442 (Oxford 2005).

W. Heurtley, Excavations at Mycenae. The grave stelai, BSA 25, 1921-1923, 126-146.

S. Hood, The Arts in Prehistoric Greece (Harmondsworth 1978).

J. Hurwit, The Dendra octopus cup and the problem of style in the fifteenth century Aegean, AJA 83, 1979, 413-426.

G. Karo, Die Schachtgräber von Mykenai (München 1930).

O. Krzyszkowska, Aegean Seals. An Introduction, BICS Suppl. 85 (London 2005).

S. Marinatos, The stelai of Circle B at Mycenae, AAA 1, 1968, 175-177.

S. Marinatos - M. Hirmer, Kreta, Thera und das mykenische Hellas ²(München 1973).

K. Müller, Frühmykenische Reliefs aus Kreta und vom griechischen Festland, JdI 30, 1915, $242-336$.

G. E. Mylonas, The figured Mycenaean stelai, AJA 55, 1951, 134-147.

S. Penner, Schliemanns Schachtgräberrund und der europäische Nordosten. Studien zur Herkunft der frühmykenischen Streitwagenausstattung (Bonn 1998).

I. Pini, Das Motiv des Löwenüberfalls in der spätminoischen und mykenischen Glyptik, in: P. Darcque - J.-C. Poursat (Hrsg.), L'iconographie minoenne. Actes de la table ronde d'Athènes (21-22 avril 1983), BCH Suppl. 11 (Athen 1985) 153-166.

I. Pini, Flechtbänder und Schlaufenbänder: eine ägäische Entwicklung oder eine Übernahme aus dem Vorderen Orient?, in: T. Korkut (Hrsg.), Anadolu'da Doğdu. Festschrift Fahri Işık (Istanbul 2004) 601-609.

J. B. Rutter, Review of Aegean Prehistory II: The prepalatial Bronze Age of the Southern and central Greek Mainland, AJA 97, 1993, 745-797.

J. G. Velsink, The ivories from the shaft graves at Mycenae, BABesch 78, 2003, 1-33.

E. T. Vermeule, The Art of the Shaft Graves of Mycenae, Lectures in Memory of Louise Taft Semple, Delivered April 30 and May 1, 1973 (Cincinnati 1975).

G. Walberg, Tradition and Innovation. Essays in Minoan Art (Mainz 1986).

H. Wingerath, Studien zur Darstellung des Menschen in der minoischen Kunst der älteren und jüngeren Palastzeit (Marburg 1995).

J. G. Younger, The stelai of Mycenae Grave Circles A and B, in: R. Laffineur - Ph. P. Betancourt (Hrsg.), TEXNH. Craftsmen, Craftswomen and Craftsmanship in the Aegean Bronze Age. Proceedings of the 6th International Aegean Conference, Philadelphia, Temple University, 18-21 April 1996, Aegaeum 16, 1 (Liège - Austin 1997) 229-239.

ao. Prof. Mag. Dr. Fritz Blakolmer

Institut für Klassische Archäologie, Universität Wien, Franz Klein-Gasse 1, A-1190 Wien

E-Mail: Fritz.Blakolmer@univie.ac.at 
Abbildungsnachweis: Abb. 1: nach Marinatos 1968, 177 Abb. 3; Abb. 2: nach Marinatos - Hirmer 1973, Taf. 220 o.; Abb. 3: nach Karo 1930, Taf. 32 u.; Abb. 4: nach Karo 1930, Taf. 32 o.; Abb. 5: mit freundlicher Genehmigung von G. St. Korres; Abb. 6: nach J. und B. Servais-Soyez, La tholos >oblongue (tombe IV) et le tumulus (tombe V) sur le Vélatouri, in: Thorikos 8, 1952/1976 (Gent 1984) 48 Abb. 26, 6a; Abb. 7: Rekonstruktionszeichnung des Verf. auf der Grundlage von Marinatos 1968, 177 Abb. 3, und Younger 1997, Taf. 89 d; Abb. 8: nach Younger 1997, Taf. 91 b; Abb. 9: nach Marinatos - Hirmer 1973, Taf. 169; Abb. 10: nach Karo 1930, Taf. 6; Abb. 11: nach Evans 1935,551 Abb. 189; Abb. 12: nach K. Demakopoulou (Hrsg.), Troja, Mykene, Tiryns, Orchomenos. Heinrich Schliemann zum 100. Todestag, Katalog (Athen 1990) 317 Abb. 275 o.; Abb. 13: nach CMS II 6 Nr. 149; Abb. 14: nach CMS II 2 Nr. 163 b; Abb. 15: nach CMS II 6 Nr. 150; Abb. 16: nach CMS II 2 Nr. 139a; Abb. 17: nach CMS II 2 Nr. 218 b; Abb. 18: nach CMS II 1 Nr. 446; Abb. 19: nach Krzyszkowska 2005, 66 Abb. 112 c; Abb. 20: nach E. $\Sigma \alpha \pi 0 v v \alpha ́-\Sigma \alpha x \varepsilon \lambda \lambda \alpha \varrho \alpha ́ x \eta$,

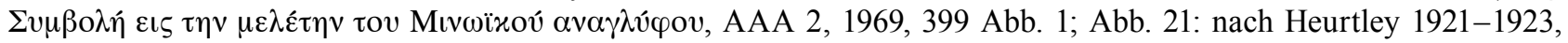
134 Abb. 30 1.; Abb. 22: nach Karo 1930, Taf. 8 1.; Abb. 23: nach J. A. Sakellarakis, The fashioning of ostrich-egg rhyta in the Creto-Mycenaean Aegean, in: D. A. Hardy u. a. (Hrsg.), Thera and the Aegean World III. Proceedings of the Third International Congress I (London 1990) 293 Abb. 33; Abb. 24: nach CMS I Nr. 227; Abb. 25: nach I. Pini, Zur >richtigen Ansicht minoisch-mykenischer Siegel- und Ringdarstellungen, in: I. Pini (Hrsg.), Fragen und Probleme der bronzezeitlichen Glyptik. Beiträge zum 3. internationalen Marburger Siegel-Symposium, 5.-7. September 1985, CMS Beih. 3 (Berlin 1989) 214 Abb. 13; Abb. 26: nach CMS V Nr. 585. 